\title{
Fixed-Time Stable Gradient Flows: Applications to Continuous-Time Optimization
}

\author{
Kunal Garg, Student Member, IEEE and Dimitra Panagou, Senior Member, IEEE
}

\begin{abstract}
Continuous-time optimization is currently an active field of research in optimization theory; prior work in this area has yielded useful insights and elegant methods for proving stability and convergence properties of the continuous-time optimization algorithms. This paper proposes novel gradientflow schemes that yield convergence to the optimal point of a convex optimization problem within a fixed time from any given initial condition for unconstrained optimization, constrained optimization, and min-max problems. It is shown that the solution of the modified gradient flow dynamics exists and is unique under certain regularity conditions on the objective function, while fixed-time convergence to the optimal point is shown via Lyapunov-based analysis. The application of the modified gradient flow to unconstrained optimization problems is studied under the assumption of gradient-dominance, a relaxation of strongconvexity. Then, a modified Newton's method is presented that exhibits fixed-time convergence under some mild conditions on the objective function. Building upon this method, a novel technique for solving convex optimization problems with linear equality constraints that yields convergence to the optimal point in fixed time is developed. More specifically, constrained optimization problems formulated as min-max problems are considered, and a novel method for computing the optimal solution in fixed-time is proposed using the Lagrangian dual. Finally, the general minmax problem is considered, and a modified scheme to obtain the optimal solution of saddle-point dynamics in fixed time is developed. Numerical illustrations that compare the performance of the proposed method against Newton's method, rescaledgradient method, and Nesterov's accelerated method are included to corroborate the efficacy and applicability of the modified gradient flows in constrained and unconstrained optimization problems.
\end{abstract}

\section{INTRODUCTION}

\section{A. Motivation}

Study of continuous-time optimization methods has been a very important part of the optimization theory from very early days [1]. Research in this area continues to this day with the aim of developing and studying differential equations that model the commonly used discrete-time optimization algorithms [2]-[4]. Establishing connections between ordinary differential equations (ODEs) and optimization has been an active topic of interest, see [4]-[6] and the references therein. The theory of ODEs offers useful insights into optimization theory and the corresponding techniques [4]; some of the notable examples, as listed in [4], include linear regression via ODEs induced by linearized Bregman iteration algorithm [7] and a continuous-time Nesterov-like accelerated algorithm

The authors are with the Department of Aerospace Engineering, University of Michigan, Ann Arbor, MI, USA; \{kgarg, dpanagou\}@umich.edu.

The authors would like to acknowledge the support of the Air Force Office of Scientific Research under award number FA9550-17-1-0284. in the context of control design [8]. The continuous-time perspective of optimization problems provides simple and elegant proofs for the convergence of solutions to the equilibrium points using Lyapunov stability theory [9]; this has led to further studies in unconstrained optimization [1], [10], constrained optimization [11], and more recently, saddle-point dynamics [5], [12]. It is worth noticing that while there is a lot of work on continuous-time optimization, most of it addresses asymptotic or exponential convergence of the solutions to the optimal point, i.e., convergence as time tends to infinity. In this paper, novel continuous-time optimization schemes are developed that possess fixed-time convergence guarantees, i.e., guarantees that the solutions of the considered ODEs converge to the optimal point of the corresponding optimization problem within a fixed time that is independent of the initial conditions.

\section{B. Gradient flows: theory and applications}

It is well known that the strict minima of a locally convex function $f: \mathbb{R}^{n} \rightarrow \mathbb{R}$ are stable equilibria of the gradient-flow (GF) dynamics $\dot{x}=-\nabla f(x)$, and that, if the sub-level sets of $f$ are compact, then the trajectories converge asymptotically to the set of critical points of $f$. In recent years, GFs have been employed in a wide range of applications, including image processing [13] and motion planning [14]. Details on design of GFs for optimization problems can be found in [10]; for an overview of convex optimization, the reader is referred to [15, Chapter 4-5].

There is a plethora of work on asymptotic convergence analysis of GF, for an overview, see [2], [3]. Recent work, for example [4], has focused on exponential stability of the GF based methods. The strong or strict convexity of the objective function is a standard assumption for exponential stability. As shown in [16], the condition can be relaxed by assuming that the objective function satisfies the Polyak-Lojasiewicz inequality (PL inequality), i.e., the objective function is gradient dominated. In [17], the authors develop cubic regularization of Newton's method with super-linear convergence rate. Other accelerated methods include Bregman-Lagrangian flows [3], where instead of standard gradient flow, Euler-Lagrange equations for the Bregman-Lagrange flow are studied for superlinear convergence.

Another set of problems where GF is used is the saddlepoint dynamics for min-max problems, where a multivariate function needs to be minimized over one set of variables and maximized over another set of variables. Saddle-point dynamics and its variations have been used extensively in the design and analysis of distributed feedback controllers [18] 
and optimization algorithms in several domains, including active power loss minimization [19], network optimization [20], and zero-sum games [21] (see [22] for a detailed presentation on various applications where saddle-point dynamics naturally arise). In [20], the authors show asymptotic stability of the saddle-point dynamics, and apply the developed methods to internet-congestion control and network-utility maximization. More recently, in [23], the authors develop general techniques for deriving minimax bounds under local differential privacy constraints. Lagrangian based primal-dual problems are another set of problems where min-max problems naturally arise. In [5], [12], the authors discuss the conditions under which the saddle-point dynamics exhibit global asymptotic convergence. In [6], [24], the authors show global exponential stability of the gradient-based method for primal-dual gradient dynamics under strong convexity-concavity assumption.

\section{Finite-time and fixed-time stability}

In the seminal work [25], the authors introduced the notion of finite-time stability (FTS), where the convergence of the solutions to the equilibrium is guaranteed within a finite time, in contrast to asymptotic or exponential convergence where the solutions converge as time goes infinity. The authors give sufficient conditions in terms of existence of a Lyapunov function for FTS. Under this notion, the settling time, or time of convergence, depends upon the initial condition. A stronger notion, called fixed-time stability (FxTS), is developed in [26], where the settling time is uniformly bounded for all initial conditions. The authors in [25], [26] discuss also the robustness of a FTS and a FxTS equilibrium, respectively; they show that the convergence properties are preserved under a class of additive vanishing disturbances, and that the trajectories of a system with a FTS or FxTS equilibrium, under nonvanishing disturbances, converge to a smaller neighborhood of the equilibrium point, as compared to a system whose equilibrium is asymptotically or exponentially stable. The primary motivation of the work in this paper is to study FxTS of the gradient-based methods with applications to convex optimization problems.

\section{Related prior work on continuous-time optimization}

In [14], the authors introduce normalized gradient flows to show finite-time convergence of the solutions to the optimal point. The authors in [27] consider convex optimization problems with equality constraints under strong convexity of the objective function, and design a discontinuous dynamics that converges to the optimal solution in finite time. Finitetime distributed optimization is studied in [28], [29], where the authors assume very specific initial conditions, such that the sum of the gradient of the objective functions is zero. In [30], the authors design a sliding-mode based technique for distributed optimization with fixed-time convergence guarantees assuming that the objective functions are strongly convex. In [31], a method of finding the optimal solution of a linear program (LP) in fixed time is proposed. In [32] and [33], the authors design finite-time converging schemes for distributed optimization where the objective function is of sum of quadratic functions and strictly convex functions, respectively.

In the aforementioned work [14], [30]-[33], the resulting dynamics are discontinuous, and the solutions are understood in the sense of Filippov. While [14] mentions that the paths traced by the discontinuous dynamics and the nominal gradient flow $\dot{x}=-\nabla f(x)$ are identical, none of the other papers show uniqueness of the solutions in forward-time for the considered discontinuous dynamics. In this work, modified gradient flows are designed with continuous dynamics, and existence and uniqueness of solutions for all times and for all initial conditions is proven. Moreover, In this paper, fixedtime convergence is considered, in contrast to the finitetime convergence in [14], [28], [29], [32], where the time of convergence grows unbounded with initial conditions.

\section{E. Contributions of the paper}

In this paper, modified GF schemes are designed for unconstrained and constrained convex optimization problems, as well as for min-max problems, with fixed-time convergence guarantees. In [28], [29], the authors assume that the Hessian of the objective function is Lipschitz continuous. This assumption as well as the strong-convexity assumption in [27], [30] are relaxed in this work, and it is shown that fixed-time convergence can be guaranteed for a larger class of problems where the objective function satisfies the PL inequality. In contrast to [14], [31], convex optimization problems with linear equality constraints are studied in this paper, and a novel method is proposed to obtain the optimal point in fixed time under certain conditions on the smoothness and convexity of the objective function. In summary, contributions of the paper are as follows:

a. FxTS-GF for unconstrained optimization: a novel GF scheme with fixed-time convergence guarantees is proposed for unconstrained convex optimization problems under gradient-dominance;

b. FxTS Newton's method for unconstrained optimization: a Newton's-based method with fixed-time convergence guarantees is developed for the minimization of a strictly-convex function;

c. FxTS-GF for constrained optimization: a novel method to solve constrained optimization problems with equality constraints in fixed time is proposed, when the conjugate of the objective function is known in closed-form;

d. FxTS saddle-point dynamics for min-max problems: a modified saddle-point dynamics with fixed-time convergence guarantees is designed for min-max problems under some mild conditions;

e. FxTS saddle-point dynamics for constrained optimization: it is shown that the proposed modified saddle-point dynamics can be used to solve constrained optimization problems when the conjugate function is not known in closed-form.

f. Numerical illustrations: various numerical examples are presented to demonstrate that the proposed method achieves super-linear, fixed-time convergence with Euler discretization. It is also demonstrated that in comparison to the nominal Newton's method, and the rescaled 
gradient method in [3], the proposed method achieves faster convergence in terms of number of iterations, while requiring lower computational (wall-clock) time, which corroborates the practical applicability of the proposed methods.

To the best of authors' knowledge, this is the first work that establishes FxTS of GF-based techniques, and demonstrates their application to nonlinear constrained optimization and saddle-point dynamics. Though the theory presented in this paper treats continuous-time dynamics, the discrete-time implementation manifests the applicability of the proposed method in practice. Recent work on rate-preserving schemes [3] and consistent-discretization schemes [34], [35] for finite or fixed-time stable dynamical systems motivates the future work of studying discretization schemes for the proposed method, which would guarantee convergence of the solutions in finite or fixed number of steps for any initial condition (see Section VI for a detailed discussion on the matter).

\section{F. Organization}

The paper is organized as follows: Section II presents an overview of the theory of FTS and FxTS, as well as an overview of convex optimization. Section III presents modified gradient flows for unconstrained optimization and constrained optimization problems with linear constraints, and fixed-time convergence to the optimal point is shown. In Section IV, the min-max problem is studied for the general saddle-point dynamics. In Section V, three numerical examples are presented to corroborate applicability of the proposed method; namely, an instance of a support-vector machine, a quadratic program, and a min-max problem. Section VI discusses the limitation of the theory of FTS or FxTS for continuous-time dynamical systems when it comes to discrete-time or discretized settings, and lays out the foundation for future work in the open areas. The conclusions and plans on future work are summarized in Section VII.

\section{BACKGROUND AND PRELIMINARIES}

\section{A. Notation}

The set of reals is denoted by $\mathbb{R}$. The Euclidean norm of $x \in \mathbb{R}^{n}$ is denote by $\|x\|$, and its transpose, by $x^{T}$. For a given function $f, f^{\star}$ denotes the optimal value of the objective function for the given optimization problem and $x^{\star}$ denotes the optimal point, i.e., $f\left(x^{\star}\right)=f^{\star}$. The conjugate of the function $f$ is denoted as $f^{*}$ and is defined as $f^{*}(y)=\sup _{x \in \mathbb{R}^{n}}\left(y^{T} x-f(x)\right)^{1}$. The notation $f \in C^{k}(U, V)$ is used for a function $f: U \rightarrow V, U \subseteq \mathbb{R}^{n}, V \subseteq \mathbb{R}^{m}$ which is $k$-times continuously differentiable, and $f \in C_{l o c}^{1,1}(U, V)$ is used to denote a continuously differentiable function whose gradient is locally Lipschitz continuous on $U$. The notation $\nabla f: \mathbb{R}^{n} \rightarrow \mathbb{R}^{n}$ and $\nabla^{2} f: \mathbb{R}^{n} \rightarrow \mathbb{R}^{n \times n}$ is used to denote the gradient and the Hessian of the function $f \in C^{2}\left(\mathbb{R}^{n}, \mathbb{R}\right)$, respectively. For a multivariate function $f \in C^{2}\left(\mathbb{R}^{n}, \mathbb{R}\right)$, the partial derivatives are denoted as $\nabla_{x_{1}} f(x) \triangleq \frac{\partial f}{\partial x_{1}}(x)$

\footnotetext{
${ }^{1}$ Note the difference between ${ }^{\star}$ for optimality and ${ }^{*}$ for the conjugate.
}

and $\nabla_{x_{1} x_{2}} f(x) \triangleq \frac{\partial^{2} f}{\partial x_{1} \partial x_{2}}(x)$, where $x_{1} \in \mathbb{R}^{r_{1}}, x_{2} \in \mathbb{R}^{r_{2}}$, $r_{1}, r_{2} \leq n$. A positive definite (respectively, semi-definite) matrix $A \in \mathbb{R}^{n \times n}$ is denoted as $A \succ 0$ (respectively, $A \succeq 0$ ); for $A, B \in \mathbb{R}^{n \times n}, A \succ B$ (respectively, $A \succeq B$ ) is used when $A-B \succ 0$ (respectively, $A-B \succeq 0$ ). For sake of brevity, the argument $x$ of a function or its derivatives is dropped, whenever clear from the context.

\section{B. Mathematical preliminaries}

First, an overview of the mathematical preliminaries and some useful results are presented. Consider the system

$$
\dot{x}=f(x),
$$

where $x \in \mathbb{R}^{n}, f: \mathbb{R}^{n} \rightarrow \mathbb{R}^{n}$ and $f(0)=0$. Assume that the solution to (1) exists, is unique, and continuous for any $x(0) \in \mathbb{R}^{n}$, for all $t \geq 0$.

Definition 1 ([25]). The origin is said to be an FTS equilibrium of (1) if it is Lyapunov stable and finite-time convergent, i.e., for all $x(0) \in \mathcal{N} \backslash\{0\}$, where $\mathcal{N}$ is some open neighborhood of the origin, $\lim _{t \rightarrow T} x(t)=0$, where $T=T(x(0))<\infty$. The origin is said to be a globally FTS equilibrium if $\mathcal{N}=\mathbb{R}^{n}$.

Here, $T$ is called as the settling time. Lyapunov conditions for FTS of the origin for system (1) are as follows.

Lemma 1 ([25]). Suppose there exists a positive definite function $V \in C^{1}(\mathcal{D}, \mathbb{R})$, where $\mathcal{D} \subset \mathbb{R}^{n}$ is a neighborhood of the origin, constants $c>0$ and $\alpha \in(0,1)$, and an open neighborhood $\mathcal{V} \subseteq \mathcal{D}$ of the origin such that

$$
\dot{V}(x)+c V(x)^{\alpha} \leq 0, \forall x \in \mathcal{V} \backslash\{0\} .
$$

Then, the origin is an FTS equilibrium of (1). Moreover, the settling time $T$ satisfies $T(x(0)) \leq \frac{V(x(0))^{1-\alpha}}{c(1-\alpha)}$.

Under the notion of FTS, the settling time $T$ depends upon the initial condition $x(0)$ and grows unbounded as $\|x(0)\|$ increases. The notion of FxTS allows the settling time to remain upper bounded, independent of the initial condition.

Definition 2 ([26]). The origin is said to be an FxTS equilibrium of (1) if it is globally FTS and the settling time $T(x(0))$ is uniformly bounded, i.e., there exists $\bar{T}<\infty$ such that $\sup _{x(0) \in \mathbb{R}^{n}} T(x(0)) \leq \bar{T}$.

Lemma 2 ([26]). Suppose there exist a positive definite function $V \in C^{1}(\mathcal{D}, \mathbb{R})$, where $\mathcal{D} \subset \mathbb{R}^{n}$ is a neighborhood of the origin, for system (1) such that

$$
\dot{V}(x) \leq-p V(x)^{\alpha}-q V(x)^{\beta}, \forall x \in \mathcal{D} \backslash\{0\},
$$

with $p, q>0,0<\alpha<1$ and $\beta>1$. Then, the origin of (1) is FxTS with settling time

$$
T \leq \frac{1}{p(1-\alpha)}+\frac{1}{q(\beta-1)} .
$$

Next, various notions of convexity, and first and second order conditions for convexity are summarized in the following lemma (see [15, Chapter 3] for more details). 
Definition 3. A function $f \in C^{1}(D, \mathbb{R})$, where $D \subset \mathbb{R}^{n}$ is a convex set, is called

- Convex if for all $x, y \in D$ and all $\alpha \in(0,1), f(\alpha x+$ $(1-\alpha) y) \leq \alpha f(x)+(1-\alpha) f(y)$;

- Concave if $(-f)$ is convex;

- Strictly convex if for all $x, y \in D$ and all $\alpha \in(0,1)$, $f(\alpha x+(1-\alpha) y)<\alpha f(x)+(1-\alpha) f(y) ;$

- m-Strongly convex if there exists $m>0$ such that $f(y) \geq f(x)+\nabla f(x)^{T}(y-x)+\frac{m}{2}\|x-y\|^{2}$, for all $x, y \in D$;

- $\beta$-Strongly smooth if for all $x, y \in D, f(y) \leq f(x)+$ $\nabla f(x)^{T}(y-x)+\frac{\beta}{2}\|x-y\|^{2}$, where $\beta>0$.

Lemma 3. First-order conditions: A function $f \in C^{1}(D, \mathbb{R})$, where $D \subset \mathbb{R}^{n}$ is a convex set, is

- Convex if and only if for all $x, y \in D, f(y) \geq f(x)+$ $\nabla f(x)^{T}(y-x)$;

- m-Strongly convex if and only if for all $x, y \in D$, $(\nabla f(x)-\nabla f(y))^{T}(x-y) \geq m\|x-y\|^{2}$ for some $m>0$.

Second-order conditions: A function $f \in C^{2}(D, \mathbb{R}), D \subset \mathbb{R}^{n}$ is

- Convex if and only if for all $x \in D, \nabla^{2} f(x) \succeq 0$;

- Strictly convex if for all $x \in D, \nabla^{2} f(x) \succ 0$;

- m-Strongly convex if and only if for all $x \in D$, $\nabla^{2} f(x) \succeq m I$ for some $m>0$.

- $\beta$-Strongly smooth if and only if for all $x \in D$, $\nabla^{2} f(x) \preceq \beta I$ for some $\beta>0$.

It follows that strong-convexity implies strict-convexity, which implies convexity.

Definition 4. A function $F: D_{1} \times D_{2} \rightarrow \mathbb{R}$, where $D_{1} \subset$ $\mathbb{R}^{n}, D_{2} \subset \mathbb{R}^{m}$, is called locally convex-concave (respectively, locally strongly or locally strictly convex-concave) if for any fixed $\bar{z} \in U_{z} \subset D_{2}, F(x, \bar{z})$ is convex (respectively, strongly or strictly convex) for all $x \in U_{x} \subset D_{1}$, and for any fixed $\bar{x} \in U_{x} \subset D_{1}, F(\bar{x}, z)$ is concave (respectively, strongly or strictly concave) for all $z \in U_{z} \subset D_{2}$.

\section{FXTS IN OPTIMIZATION}

In this section, novel gradient flow schemes are proposed for unconstrained and constrained convex optimization problems. First, unconstrained optimization problems are considered.

\section{A. Unconstrained optimization: FTS scheme}

Consider the unconstrained minimization problem

$$
\min _{x \in \mathbb{R}^{n}} f(x),
$$

where $f: \mathbb{R}^{n} \rightarrow \mathbb{R}$. The following assumption is made about the problem (5).

Assumption 1. The minimum value of $f(x)$ is attained, i.e., there exists $x^{\star} \in \mathbb{R}^{n}$ such that $-\infty<f^{\star}=f\left(x^{\star}\right)$.

Remark 1. For (5), Assumption 1 is a necessary condition for convergence of gradient-based methods to an optimal solution. Coercivity, or equivalently, compactness of the sub-level sets of the convex function $f$ is a sufficient condition to guarantee existence of a minimizer [36, Chapter 2].
Lemma 4 ([15]). If $f$ is convex and differentiable, then $a$ point $x^{\star}$ is the global optimal point of the function $f$ if and only if $\nabla f\left(x^{\star}\right)=0$. Furthermore, if $f$ is strictly convex, then the optimal point $x^{\star}$ is unique.

There has been a lot of research on developing discrete-time optimization schemes with convergence rate faster than linear (see [3], [17] and references therein). The continuous variant of such discrete-time schemes are also studied by various authors. In [3], the authors discuss the following scheme

$$
\dot{x}=-\frac{\nabla f(x)}{\|\nabla f(x)\|^{\frac{p-2}{p-1}}},
$$

where $p>2$ as a modification of GF. It is shown that the convergence rate for the solutions of (6) is given as

$$
f(x(t))-f^{\star} \leq O\left(\frac{1}{t^{p-1}}\right),
$$

under the assumption that the level-sets of $f(x)$ are bounded. The flow in (6) is referred to as rescaled GF in the subsequent text. In this subsection, it is shown that the optimal point of (5) is actually an FTS equilibrium of (6). Then, in the subsequent subsections, modifications of (6) are presented with fixed-time convergence guarantees.

Theorem 1. If $f \in C^{2}\left(\mathbb{R}^{n}, \mathbb{R}\right)$ is $k$-strongly convex for some $k>0$, then the trajectories of (6) converge to the optimal point $x^{\star}$ in finite time $T=T(x(0))$, for any $p>2$.

Proof: First, using Lemma 3, one has that strong convexity of $f$ implies that the optimal solution $x^{\star}$ of (5) is unique and satisfies $\nabla f\left(x^{\star}\right)=0$. Choose $V(x)=\frac{1}{2}\|\nabla f(x)\|^{2}$ as the candidate Lyapunov function. $k$-strong convexity of $f$ implies that $\nabla^{2} f(x) \succeq k I$ for all $x \in \mathbb{R}^{n}$. Using this, one obtains that the time derivative of $V$ along (6) satisfies

$$
\begin{aligned}
\dot{V} & =\nabla f^{T} \nabla^{2} f \dot{x}=-\nabla f^{T} \nabla^{2} f \frac{\nabla f}{\|\nabla f\|^{\frac{p-2}{p-1}}} \\
& \leq-k \nabla f^{T} \frac{\nabla f}{\|\nabla f\|^{\frac{p-2}{p-1}}}=-k\|\nabla f\|^{2-\frac{p-2}{p-1}} \\
& =-k\|\nabla f\|^{\frac{p}{p-1}}=-k 2^{\frac{p}{2(p-1)}} V^{\frac{p}{2(p-1)}} .
\end{aligned}
$$

Define $k_{1}=k 2^{\frac{p}{2(p-1)}}>0$ and $\beta_{1}=\frac{p}{2(p-1)}$, so that $0<\beta_{1}<$ 1 , and one obtains $\dot{V} \leq-k_{1} V^{\beta_{1}}$. Using Lemma 1, one obtains that $\|\nabla f(x(t))\|=0$ for all $t \geq T$ where $T \leq \frac{V(x(0))^{1-\beta_{1}}}{k_{1}\left(1-\beta_{1}\right)}$. Since the function is strongly convex, the sublevel sets of the norm of $\nabla f$ are bounded. Thus, $V$ is radially unbounded, and hence, the result holds for all $x(0) \in \mathbb{R}^{n}$.

Remark 2. For a given $p>2$, denote the bound on the time of convergence as $\bar{T}_{p}$. As noted in [3], the limiting case of (6) as $p \rightarrow \infty$, called normalized GF, is studied in [14], and it is shown that the time of convergence is upper bounded by $\frac{1}{k}\|\nabla f(x(0))\|$ under the assumption of strong-convexity. The same bound on the time of convergence is recovered by allowing $p \rightarrow \infty$ in the bound of the settling time $\bar{T}_{\infty}$ in Theorem 1. Note that for initial values farther away from the optimal point, the upper bound on the time of convergence satisfy $\bar{T}_{p} \leq \bar{T}_{\infty}$, i.e., the upper bound for $2<p<\infty$ is lower than $\frac{1}{k} \| \nabla f(x(0) \|$. 
It is clear from the expression of the bound on the settling time $T$ in Theorem 1 that it grows unbounded as the distance of $x(0)$ increases from the optimal point $x^{\star}$. Inspired from (6) and noting its finite-time convergence guarantees, a modified $\mathrm{GF}$ is designed in this subsection with fixed-time convergence guarantees, so that the the optimal point of (5) can be obtained within a fixed time for any $x(0) \in \mathbb{R}^{n}$.

\section{B. Unconstrained optimization: FxTS-GF scheme}

Consider the flow equation

$$
\dot{x}=-c_{1} \frac{\nabla f(x)}{\|\nabla f(x)\|^{\frac{p_{1}-2}{p_{1}-1}}}-c_{2} \frac{\nabla f(x)}{\|\nabla f(x)\|^{\frac{p_{2}-2}{p_{2}-1}}},
$$

where $c_{1}, c_{2}>0, p_{1}>2$ and $1<p_{2}<2$. In what follows, (8) is referred to as FxTS-GF. First, it is shown that the equilibrium points of the right-hand side of (8) are critical points $^{2}$ of the function $f$, and that the dynamics in (8) is continuous for all $x \in \mathbb{R}^{n}$. $^{3}$

Lemma 5. A point $\bar{x} \in \mathbb{R}^{n}$ is an equilibrium point of (8) if and only if $\|\nabla f(\bar{x})\|=0$.

Lemma 6. If $f \in C_{l o c}^{1,1}\left(\mathbb{R}^{n}, \mathbb{R}\right)$, then the right-hand side of (8) is continuous for all $x \in \mathbb{R}^{n}$.

Proofs of Lemma 5 and Lemma 6 are given in Appendix A, $\mathrm{B}$, respectively. Next, it is shown that the solutions of (8) exist and are unique in forward time.

Proposition 1. If the function $f \in C_{l o c}^{1,1}\left(\mathbb{R}^{n}, \mathbb{R}\right)$ is convex with unique minimizer, then for any $x(0) \in \mathbb{R}^{n}$, the solution of (8) exists and is unique for all $t \geq 0$.

Proof: The result [37, Proposition 2] shows existence and uniqueness of solutions for an ODE of the form (8) with $X=\nabla f$. Per the statement of the Theorem, $f \in C_{l o c}^{1,1}$, which implies that $\nabla f$ is locally Lipschitz. Also, with $0<\alpha_{1}=$ $1-\frac{p_{1}-2}{p_{1}-1}<1$ and $\alpha_{2}=1-\frac{p_{2}-2}{p_{2}-1}>1$, all the conditions of [37, Proposition 2] are satisfied. Hence, the solution of (8) exists in forward time and is unique, for any initial condition $x(0) \in \mathbb{R}^{n}$.

Before presenting the main result of this subsection, the following required assumptions on the objective function $f$ is made.

Assumption 2. (Gradient dominated) The function $f \in$ $C_{\text {loc }}^{1,1}\left(\mathbb{R}^{n}, \mathbb{R}\right)$ has a unique minimizer $x=x^{\star}$ and satisfies the Polyak-Łojasiewicz (PL) inequality, or is gradient dominated, with $\mu_{f}>0$, i.e., for all $x \in \mathbb{R}^{n}$,

$$
\frac{1}{2}\|\nabla f(x)\|^{2} \geq \mu_{f}\left(f(x)-f^{\star}\right) .
$$

Remark 3. Strong convexity of the objective function is a standard assumption used in literature to show exponential convergence for gradient flows. As noted in [16], PL inequality is the weakest condition among other similar conditions popularly used in the literature to show linear convergence

\footnotetext{
${ }^{2}$ Recall that a point $x$ is called a critical point of a $C^{1}$ function $f$ if $\nabla f(x)=0$.

${ }^{3}$ Note that the dynamics in (8) is defined as $\dot{x}=0$ when $\nabla f(x)=0$.
}

in discrete-time (exponential, in continuous-time) gradientbased algorithms. Particularly, a strongly convex function $f \in C^{1}\left(\mathbb{R}^{n}, \mathbb{R}\right)$ satisfies PL inequality. Note that under this assumption, it is not required for the objective function $f$ to be convex.

It is shown in [16, Theorem 2] that satisfaction of PL inequality implies that the function $f$ has quadratic growth, i.e.,

$$
f(x)-f^{\star} \geq \frac{\mu_{f}}{2}\left\|x-x^{\star}\right\|^{2} .
$$

for all $x$, where $\mu_{f}$ is as defined in (9). The following result can now be stated.

Theorem 2. If the objective function $f$ satisfies Assumptions 1 and 2, then, the trajectories of (8) converge to the optimal point $x^{\star}$ in fixed time for all $x(0)$.

Proof: First, note that the solutions of (8) exist and are unique for all $x(0) \in \mathbb{R}^{n} \backslash\left\{x^{\star}\right\}$ [37, Proposition 2]. Note that the convexity assumption in [37, Proposition 2], and consequently, in Proposition 1, is sufficient to show uniqueness of the solutions starting from the minimizer, i.e., for the case when $x(0)=x^{\star}$. For the case when the function $f$ is not convex, the uniqueness of the solution can still be guaranteed if the equilibrium point is attractive. Assume that for (8) with a non-convex $f$, there exist two solutions, namely the trivial solution $x_{1}(\cdot)=x^{\star}$, and another solution such that $x_{2}(\cdot) \neq x^{\star}$, with $x_{1}(0)=x_{2}(0)=x^{\star}$. Define $V_{1}=f\left(x_{1}(t)\right)-f\left(x_{2}(t)\right)=f\left(x_{1}(t)\right)-f^{\star}$ as a candidate Lyapunov function. Note that since $x_{1}(t) \neq x^{\star}$, $f\left(x_{1}(t)\right)>f^{\star}$, and hence, $V_{1}$ is a positive definite function. The time derivative of $V_{1}$ reads $\dot{V}_{1}=\nabla f\left(x_{1}(t)\right)^{T} \dot{x}_{1}(t)$, which along the trajectories of (8) satisfies $\dot{V}_{1} \leq 0$. Since $V_{1}(0)=f\left(x_{1}(0)\right)-f\left(x_{2}(0)\right)=0$, and $\dot{V}_{1} \leq 0$, using [38, Theorem 3.15.1], one obtains that $x_{1}(t)=x_{2}(t)=x^{\star}$, i.e., the solution of (8) is unique for $x(0)=x^{\star}$.

Now, consider the candidate Lyapunov function $V(x)=$ $\frac{1}{2}\left(f(x)-f^{\star}\right)^{2}$. From (10), it is clear that $V$ is radially unbounded. The time derivative along the trajectories of (8) reads

$$
\begin{aligned}
\dot{V} & =\left(f-f^{\star}\right)(\nabla f)^{T}\left(-c_{1} \frac{\nabla f}{\|\nabla f\|^{\frac{p_{1}-2}{p_{1}-1}}}-c_{2} \frac{\nabla f}{\|\nabla f\|^{\frac{p_{2}-2}{p_{2}-1}}}\right) \\
& =-c_{1}\left(f-f^{\star}\right)\|\nabla f\|^{2-\frac{p_{1}-2}{p_{1}-1}}-c_{2}\left(f-f^{\star}\right)\|\nabla f\|^{2-\frac{p_{2}-2}{p_{2}-1}} \\
& =-c_{1}\left(f-f^{\star}\right)\|\nabla f\|^{\alpha_{1}}-c_{2}\left(f-f^{\star}\right)\|\nabla f\|^{\alpha_{2}} \\
& \stackrel{(9)}{\leq}-c_{1}\left(2 \mu_{f}\right)^{\frac{\alpha_{1}}{2}}\left(f-f^{\star}\right)^{1+\frac{\alpha_{1}}{2}}-c_{2}\left(2 \mu_{f}\right)^{\frac{\alpha_{2}}{2}}\left(f-f^{\star}\right)^{1+\frac{\alpha_{2}}{2}} \\
& =-c_{1} 2^{\frac{2+3 \alpha_{1}}{4}} \mu_{f}^{\frac{\alpha_{1}}{2}} V^{\frac{2+\alpha_{1}}{4}}-c_{2} 2^{\frac{2+3 \alpha_{2}}{4}} \mu_{f}^{\frac{\alpha_{2}}{2}} V^{\frac{2+\alpha_{2}}{4}} \\
& =-k_{1} V^{\frac{2+\alpha_{1}}{4}}-k_{2} V^{\frac{2+\alpha_{2}}{4}},
\end{aligned}
$$

where $\alpha_{1}=2-\frac{p_{1}-2}{p_{1}-1}, \alpha_{2}=2-\frac{p_{2}-2}{p_{2}-1}, k_{1}=c_{1} 2^{\frac{2+3 \alpha_{1}}{4}} \mu_{f}^{\frac{\alpha_{1}}{2}}$ and $k_{2}=c_{2} 2^{\frac{2+3 \alpha_{2}}{4}} \mu_{f}^{\frac{\alpha_{2}}{2}}$. Since $\alpha_{1}<2$ and $\alpha_{2}>2$, one has $\frac{2+\alpha_{1}}{4}<1$ and $\frac{2+\alpha_{2}}{4}>1$. Hence, from Lemma 2, one obtains that for $t \geq T_{1}, f(x(t))=f^{\star}$, which is equivalent to $x(t)=$ $x^{\star}$ under Assumption 2, where $T_{1} \leq \frac{4}{k_{1}\left(2-\alpha_{1}\right)}+\frac{4}{k_{2}\left(\alpha_{2}-2\right)}$. Hence, the trajectories of (8) converge to the optimal point $x^{\star}$ of (5) in fixed time. 
Remark 4. Note that the difference between the proposed modified gradient flow (8) and the rescaled gradient flow (6) is the second term with exponent $1<p_{2}<2$. This term results into the second term $-V^{\beta}$ in (3), while the first term, with exponent $p_{1}>2$, results into the first term $-V^{\alpha}$ in (3). Intuitively, compared to the exponential convergence condition $\dot{V} \leq-V$, terms $V^{\beta}$ and $V^{\alpha}$ dominate the term $V$ when $V$ is large and small, respectively, resulting into accelerated convergence for both small and large initial distance from the equilibrium point. Since (6) contains only the first term, which dominates when $V$ is small, the time of convergence, though finite, grows larger as the initial distance from the equilibrium increases.

It is showed that the FxTS-GF in (8) can be used to find the optimal solution of (5) in fixed time. As mentioned in Remark 3 , Assumption 2 is a relaxation used to show exponential convergence of gradient flow. Hence, all such problems which have been shown to have exponential convergence under strong-convexity can be solved within fixed time using (8). It is easy to show that if a function $f: \mathbb{R}^{m} \rightarrow \mathbb{R}$ is strongly convex, then the function $g: \mathbb{R}^{n} \rightarrow \mathbb{R}$, defined as $g(x)=f(A x)$, $A \in \mathbb{R}^{n \times m}$, is strongly convex if $A$ is full row-rank. If matrix $A$ is not full row-rank, then $g$ may not be strongly convex. On the other hand, as shown in [16, Appendix 2.3], $g$ still satisfies PL inequality for any matrix $A$. Below, an example of an important class of problems is given for which, the objective function satisfies PL inequality (see [16] for more examples on useful functions that satisfy PL inequality).

Example 1. Least squares: Consider the optimization problem

$$
\min _{x \in \mathbb{R}^{n}} f(A x)=\|A x-b\|^{2},
$$

where $x \in \mathbb{R}^{n}, A \in \mathbb{R}^{n \times n}$ and $b \in \mathbb{R}^{n}$. Here, the function $f(x)=\|x-b\|^{2}$ is strongly-convex, and hence, $g(x)=$ $\|A x-b\|^{2}$ satisfies PL inequality for any matrix A. Linear regression: Consider the optimization problem

$$
\min _{x \in \mathbb{R}^{n}} f(A x)=\sum_{i=1}^{m} \log \left(1+b_{i} a_{i}^{T} x\right),
$$

where $x \in \mathbb{R}^{n}, a_{i} \in \mathbb{R}^{n}$ and $b \in \mathbb{R}$ for $i=1,2, \ldots, m$. Here, the function $g(x)=f(A x)$ satisfies PL inequality for any matrix A.

The objective functions in (11) and (12) satisfy PL inequality, but need not be strongly convex for any matrix $A$; if additionally, uniqueness of the optimal solutions of (11) and (12) is assumed, one can use (8) to find the optimal solutions for (11) and (12), respectively, in fixed time. These are important classes of functions in machine learning problems. Next, the modification of the Newton's method based GF is presented to guarantee FxTS for a class of functions that do not satisfy Assumption 2.

\section{FxTS Newton's method}

In this subsection, a modified Newton's method is proposed that guarantees fixed-time convergence to the optimal point.
The nominal Newton's method is defined as

$$
\dot{x}=-\left(\nabla^{2} f(x)\right)^{-1} \nabla f(x) .
$$

It is well-known that under certain conditions on the function $f$, (13) can achieve exponential convergence. The following assumption is made about the objective function $f$.

Assumption 3. The function $f \in C^{2}\left(\mathbb{R}^{n}, \mathbb{R}\right)$ is strictly convex.

Per Assumption 3, $\nabla^{2} f \succ 0$, which implies that the Hessian is invertible ${ }^{4}$, and with Assumption 1, using Lemma 4, one has that the optimal point $x^{\star}$ is unique. Note that if $f$ satisfies Assumption 3, it is not necessary that it satisfies Assumption 2. So, for (5) with this class of functions, fixed-time convergence cannot be guaranteed using (8). Hence, another modified GF is proposed so that fixed-time convergence for this class of functions can be guaranteed. Consider the flow equation for FxTS Newton's method

$$
\dot{x}=-\left(\nabla^{2} f(x)\right)^{-1}\left(c_{1} \frac{\nabla f(x)}{\|\nabla f(x)\|^{\frac{p_{1}-2}{p_{1}-1}}}+c_{2} \frac{\nabla f(x)}{\|\nabla f(x)\|^{\frac{p_{2}-2}{p_{2}-1}}}\right),
$$

where $c_{1}, c_{2}>0, p_{1}>2$ and $1<p_{2}<2$. The following result can now be stated.

Theorem 3. If $f$ satisfies Assumptions 1 and 3, then the trajectories of (14) converge to the optimal point $x^{\star}$ in fixed time $T_{N M}$ for any initial condition $x(0) \in \mathbb{R}^{n}$.

Proof: Note that under Assumption 3, per Proposition 1 , solutions of (14) exist and are unique for all $x(0) \in \mathbb{R}^{n}$. Consider the Lyapunov function $V(x)=\frac{1}{2}\|\nabla f(x)\|^{2}$. Since the sub-level sets of norm of its gradient $\nabla f$ are bounded for a strictly convex function under Assumption 1 ( [39, Corollary 8.7.1]), the candidate Lyapunov function $V$ is radially unbounded. The time derivative of this function along the trajectories of (14) reads

$$
\begin{aligned}
\dot{V} & =(\nabla f)^{T}\left(\nabla^{2} f\right) \dot{x} \\
& =-(\nabla f)^{T}\left(c_{1} \frac{\nabla f}{\|\nabla f\|^{\frac{p_{1}-2}{p_{1}-1}}}+c_{2} \frac{\nabla f}{\|\nabla f\|^{\frac{p_{2}-2}{p_{2}-1}}}\right) \\
& =-c_{1}\|\nabla f\|^{2-\frac{p_{1}-2}{p_{1}-1}}-c_{2}\|\nabla f\|^{2-\frac{p_{2}-2}{p_{2}-1}} \\
& \leq-c_{1} 2^{\frac{\alpha_{1}}{2}} V^{\frac{\alpha_{1}}{2}}-c_{2} 2^{\frac{\alpha_{2}}{2}} V^{\frac{\alpha_{2}}{2}},
\end{aligned}
$$

where $\alpha_{1}=2-\frac{p_{1}-2}{p_{1}-1}$ and $\alpha_{2}=2-\frac{p_{2}-2}{p_{2}-1}$. Since $p_{1}>2$ and $1<p_{2}<2$ one obtains that $1<\alpha_{1}<2$ and $\alpha_{2}>2$. Hence, using Lemma 2, one obtains that the trajectories of (14) converge to the optimal point $x^{\star}$ in the fixed time $T_{N M}$

for all $x(0) \in \mathbb{R}^{n}$, where $T_{N M} \leq \frac{2^{1-\frac{\alpha_{1}}{2}}}{c_{1}\left(2-\alpha 1_{1}\right)}+\frac{2^{1-\frac{\alpha_{2}}{2}}}{c_{2}\left(\alpha_{2}-2\right)}$.
$\quad$ While strongly-convex functions satisfy PL inequality [16], strictly convex functions do not satisfy PL inequality in general. So, for convex optimization problems with strictly convex objective functions, that do not satisfy Assumption 2, (14) can be used to find the optimal solution of (5) within a fixed time. One example is the class of quartic functions, which can be used to reformulate standard QP with sign constraints as uncontrained optimization problem [40].

\footnotetext{
${ }^{4}$ This is needed so that the right-hand side in the Newton's method is welldefined.
} 
Example 2. Consider the optimization problem

$$
\begin{aligned}
& \min _{x} x^{T} Q x+c^{T} x, \\
& \text { s.t. } x_{i} \geq 0, i=1,2, \ldots, n,
\end{aligned}
$$

where $x, c \in \mathbb{R}^{n}$ and $Q \in \mathbb{R}^{n \times n}$ is a positive definite matrix. Let $z \in \mathbb{R}^{n}$ be defined as $x_{i}=z_{i}^{2}$, to get rid of the sign contraints, and re-write (15) in terms of $z$

$$
\min _{z} z^{T} Z Q Z z+c^{T} Z z
$$

where $Z \in \mathbb{R}^{n \times n}$ is a diagonal matrix consisting of elements $z_{i}$, i.e.,

$$
Z_{i j}= \begin{cases}z_{i}, & i=j \\ 0, & i \neq j,\end{cases}
$$

for $i, j=1,2, \ldots, n$. The optimal solution $\bar{x}$ of (15) is given by $\bar{x}_{i}=\bar{z}_{i}^{2}$, where $\bar{z}$ is the optimal solution of (16).

It is clear that the objective function in (16) is a quartic, is not strongly convex and may not satisfy PL inequality. Nevertheless, it is strictly convex and hence, (14) can be used to find the optimal point of (16) within fixed time.

Upto now, unconstrained minimization problems have been considered. Next, constrained minimization problems are studied, and FxTS-GF based methods are proposed with fixed-time convergence guarantees.

\section{Convex optimization with linear equality constraints}

Consider the optimization problem

$$
\begin{aligned}
& \min _{x \in \mathbb{R}^{n}} f(x), \\
& \text { s.t. } A x=b,
\end{aligned}
$$

where $f: \mathbb{R}^{n} \rightarrow \mathbb{R}$ is convex, $A \in \mathbb{R}^{m \times n}$ and $b \in \mathbb{R}^{m}$.

Assumption 4. The matrix $A$ is full row-rank and the objective function $f$ is coercive.

Remark 5. Assumption 4 is commonly used in constrained optimization [24]; the matrix A being full row-rank guarantees that the feasible set is non-empty and closed, and thus, coercivity of the convex function $f$ guarantees that the solution of (17) exists [36, Chapter 2].

Define $g: \mathbb{R}^{m} \rightarrow \mathbb{R}$ as

$$
g(\nu)=\inf _{x \in \mathbb{R}^{n}}\left(f(x)+\nu^{T}(A x-b)\right),
$$

so that the dual problem (see [15, Chapter 5]) for (17) is given by

$$
\sup _{\nu \in \mathbb{R}^{m}} g(\nu)
$$

Using (18), rewrite (19) as

$$
\sup _{\nu \in \mathbb{R}^{m}} \inf _{x \in \mathbb{R}^{n}} L(x, \nu) \triangleq f(x)+\nu^{T}(A x-b) .
$$

It is clear that (20) is a saddle-point problem, where the function $L(x, \nu)$ needs to be minimized over $x$ and maximized over $\nu$. Using (18), one obtains ([15, Section 5.1])

$$
g(\nu)=-\nu^{T} b-f^{*}\left(-A^{T} \nu\right),
$$

where $f^{*}: \mathbb{R}^{n} \rightarrow \mathbb{R}$ is the conjugate of $f .{ }^{5}$ Note that the

\footnotetext{
${ }^{5}$ Since the considered space is the finite-dimensional vector space $\mathbb{R}^{n}$ with the Euclidean norm, the dual space is $\mathbb{R}^{n}$ with the dual norm $\|\cdot\|_{*}=\|\cdot\|$.
}

function $f^{*}$ is always convex, whether $f$ is convex or not $[15$, Chapter 3]. It can be readily seen from (21) that $g$ is a concave function (since $f^{*}$ is convex, $-f^{*}$ is concave). As shown in [41, Section 3.5], strong-convexity of function $f$ and strongsmoothness of its conjugate $f^{*}$ are equivalent. Using this, the following results can be stated.

Lemma 7. If $f$ is a convex, $\beta$-strongly smooth function, then the function $g$ defined as per (18) is $\alpha$-strongly concave, for some $\alpha>0$.

The proof is provided in Appendix C. Thus, the following assumption on $f$ is made.

Assumption 5. The objective function $f \in C_{\text {loc }}^{1,1}\left(\mathbb{R}^{n}, \mathbb{R}\right)$ is $\beta_{1}$-strongly convex, $\beta_{2}$-strongly smooth, its conjugate function $f^{*}$ is known in closed-form, and satisfies $f^{*} \in C_{\text {loc }}^{1,1}\left(\mathbb{R}^{n}, \mathbb{R}\right)$.

See Remark 7 and Corollary 2 for the case when $f^{*}$ is not known in closed-form. Consider the dynamical system

$$
\dot{\nu}=-c_{1} \frac{-\nabla g(\nu)}{\|\nabla g(\nu)\|^{\frac{p_{1}-2}{p_{1}-1}}}-c_{2} \frac{-\nabla g(\nu)}{\|\nabla h(\nu)\|^{\frac{p_{2}-2}{p_{2}-1}}},
$$

with $c_{1}, c_{2}>0, p_{1}>2$ and $1<p_{2}<2$. Note that the assumptions on functions $f, f^{*}$, and matrix $A$ implies $\sup \inf _{x} L(x, \nu)=\inf _{x} \sup L(x, \nu)$ ([15, Section 5.5]). Also, using Proposition 1 , one has that the solutions of (22) exist and are unique for all $\nu(0) \in \mathbb{R}^{m}$.

Lemma 8. The trajectories of (22) reach the optimal point $\nu^{\star}$ of (19) in fixed time $T_{\nu}$ for all initial conditions $\nu(0) \in \mathbb{R}^{m}$.

Proof: Per Lemma 7, $-g(\nu)$ is $\alpha$-strongly convex. Thus, $g$ satisfies PL inequality (9) with some constant $\mu_{g}>0$. Since $g$ is strongly convex, and the maximizer $\nu^{\star}$ of $g$ exists, it is also unique. This implies that $g$ satisfies Assumptions 1 and 2. Hence, using Theorem 2, one obtains that the trajectories of (22) reach the the maximizer $\nu^{\star}$ of $g(\nu)$ in a fixed time $T_{\nu} \leq \frac{4}{k_{3}\left(2-\alpha_{1}\right)}+\frac{4}{k_{4}\left(\alpha_{2}-2\right)}$ for all initial conditions $\nu(0)$, where $\alpha_{1}=2-\frac{p_{1}-2}{p_{1}-1}, \alpha_{2}=2-\frac{p_{2}-2}{p_{2}-1}, k_{3}=c_{1} 2^{\frac{2+3 \alpha_{1}}{4}} \mu_{g}^{\frac{\alpha_{1}}{2}}$ and $k_{4}=$ $c_{2} 2^{\frac{2+3 \alpha_{2}}{4}} \mu_{g}^{\frac{\alpha_{2}}{2}}$.

Under the assumption of existence (Assumption 4) and uniqueness (guaranteed by Assumption 5) of the optimal point of (17) and using the fact that $-g(\nu)$ is $\alpha$-strongly convex, one obtains that the minimizer of $L\left(x, \nu^{\star}\right)$ is the optimal solution of (17) [15, Section 5.5.5]. Using this, one obtains that

$$
x^{\star}=\arg \min _{x \in \mathbb{R}^{n}} L\left(x, \nu^{\star}\right)
$$

or, in other words, $x^{\star}$ satisfies $\nabla_{x} L\left(x^{\star}, \nu^{\star}\right) \triangleq \nabla f\left(x^{\star}\right)+$ $\nu^{* T} A=0$. Hence, the trajectories of the system

$$
\dot{x}=-d_{1} \frac{\nabla_{x} L\left(x, \nu^{\star}\right)}{\left\|\nabla_{x} L\left(x, \nu^{\star}\right)\right\|^{\frac{q_{1}-2}{q_{1}-1}}}-d_{2} \frac{\nabla_{x} L\left(x, \nu^{\star}\right)}{\left\|\nabla_{x} L\left(x, \nu^{\star}\right)\right\|^{\frac{q_{2}-2}{q_{2}-1}}},
$$

with $d_{1}, d_{2}>0, q_{1}>2$ and $1<q_{2}<2$, converge to the optimizer of (17). The following result can now be stated.

Theorem 4. Let Assumptions 4 and 5 hold. Then, the optimal point $x^{\star}$ of (17) can be found in fixed time $T_{e q}$ by first solving 
(22) for any $\nu(0) \in \mathbb{R}^{m}$, and then, solving (24) for any $x(0) \in$ $\mathbb{R}^{n}$, with

$T_{e q} \leq \frac{4}{k_{3}\left(2-\alpha_{1}\right)}+\frac{4}{k_{4}\left(\alpha_{2}-2\right)}+\frac{4}{k_{5}\left(2-\alpha_{3}\right)}+\frac{4}{k_{6}\left(\alpha_{4}-2\right)}$ where $k_{i}, \alpha_{i}$ are functions of $c_{1}, c_{2}, d_{1}, d_{2}, p_{1}, p_{2}, q_{1}, q_{2}$.

Proof: From Lemma 8, one obtains that the trajectories of (22) reach the optimizer $\nu^{\star}$ of (19) in fixed time $T_{\nu} \leq \frac{4}{k_{3}\left(2-\alpha_{1}\right)}+\frac{4}{k_{4}\left(\alpha_{2}-2\right)}$. Now, since $f(x)$ is strongly convex, it follows that $L(\cdot, \nu)$ is strongly convex for each $\nu \in \mathbb{R}^{m}$, and in particular, $L\left(\cdot, \nu^{\star}\right)$ is strongly convex, and hence, also satisfies PL inequality for some constant $\mu_{L}>0$. Furthermore, it can be easily shown that $L\left(\cdot, \nu^{\star}\right)$ satisfies Assumptions 1 and 2. Therefore, from Theorem 2, one has that there exists a fixed time $T_{x}$ such that the trajectories of (24) reach the optimal point of (23) in $T_{x} \leq \frac{4}{k_{5}\left(2-\alpha_{3}\right)}+\frac{4}{k_{6}\left(\alpha_{4}-2\right)}$ for all initial conditions $x(0)$, where $\alpha_{3}=2-\frac{q_{1}-2}{q_{1}-1}, \alpha_{4}=$ $2-\frac{q_{2}-2}{q_{2}-1}, k_{5}=d_{1} 2^{\frac{2+3 \alpha_{3}}{4}} \mu_{L}^{\frac{\alpha_{1}}{2}}$ and $k_{6}=d_{2} 2^{\frac{2+3 \alpha_{4}}{4}} \mu_{L}^{\frac{\alpha_{1}}{2}}$. Hence, one has that the optimal point of (17) can be obtained in fixed time $T_{e q} \leq T_{x}+T_{\nu}$, by first solving (22) and then, (24).

A very important class of optimization problems in machine learning and model predictive control (MPC) is the class of quadratic programs (QPs). In the following example, it is shown that QPs with equality constraints that satisfy Assumption 5 fit into the proposed framework.

Example 3. Consider the following $Q P$ with equality constraints

$$
\begin{aligned}
& \min _{x \in \mathbb{R}^{n}} \frac{1}{2} x^{T} Q x+c^{T} x, \\
& \text { s.t. } A x=b,
\end{aligned}
$$

where $Q \in \mathbb{R}^{n \times n}$ is positive definite and $A \in \mathbb{R}^{m \times n}$ has full row-rank. The function $g(\nu)$ for (25) is given as

$$
\begin{aligned}
g(\nu) & =\inf _{x \in \mathbb{R}^{n}}\left(\frac{1}{2} x^{T} Q x+c^{T} x+\nu^{T}(A x-b)\right) \\
& =-\nu^{T} b-\frac{1}{2}\left(c-A^{T} \nu\right)^{T} Q^{-1}\left(c-A^{T} \nu\right) .
\end{aligned}
$$

Hence, one has that $f^{*}\left(-A^{T} \nu\right)=-\frac{1}{2}\left(c-A^{T} \nu\right)^{T} Q^{-1}(c-$ $\left.A^{T} \nu\right)$. It can be readily verified that the functions $f, f^{*}$ satisfy Assumption 5.

Remark 6. The assumption on locally Lipschitz continuity of the gradient of $f$ or $f^{*}$ (in Assumptions 2 and 5) is sufficient for uniqueness of the solution of the concerned modified GF. This assumption can be further relaxed by using [38, Theorem 3.15.11], where only continuity of the vector field is shown to be sufficient for existence and uniqueness of the solutions. Furthermore, using [41, Theorem 3.5.10], one obtains that $f^{*} \in C^{1}$ if $f$ is strongly convex. Thus, Assumption 5 (as well as Assumption 2) can be relaxed by allowing $f \in C^{1}$ and imposing no additional assumptions on $f^{*}$.

\section{FXTS OF SADDLE-POINT DyNAMICS}

In this section, min-max problems are considered that can be formulated as saddle-point dynamics, and a modification is studied so that optimal point, which is a saddle-point, can be found within a fixed time. To this end, the general saddlepoint problem of minimizing a function $F(x, z)$ over $x \in \mathbb{R}^{n}$ and maximizing over $z \in \mathbb{R}^{m}$ is considered, where $F: \mathbb{R}^{n} \times$ $\mathbb{R}^{m} \rightarrow \mathbb{R}$. Formally, this can be stated as

$$
\max _{z \in \mathbb{R}^{m}} \min _{x \in \mathbb{R}^{n}} F(x, z) .
$$

A point $\left(x^{\star}, z^{\star}\right)$ is called as local saddle-point of $F$ (as well as local optimal solution of (26)), if there exist open neighborhoods $U_{x} \subset \mathbb{R}^{n}$ and $U_{z} \subset \mathbb{R}^{m}$ of $x^{\star}$ and $z^{\star}$, respectively, such that for all $(x, z) \in U_{x} \times U_{z}$, one has

$$
F\left(x^{\star}, z\right) \leq F\left(x^{\star}, z^{\star}\right) \leq F\left(x, z^{\star}\right) .
$$

The point $\left(x^{\star}, z^{\star}\right)$ is global saddle-point if $U_{x}=\mathbb{R}^{n}$ and $U_{z}=\mathbb{R}^{m}$. The following assumption is made.

Assumption 6. A saddle point $\left(x^{\star}, z^{\star}\right)$ exists that solves (26). Furthermore, the function $F \in C^{2}\left(\mathbb{R}^{n} \times \mathbb{R}^{m}, \mathbb{R}\right)$ is locally strictly convex-concave in an open neighborhood $U \subset$ $\mathbb{R}^{n} \times \mathbb{R}^{m}$ of the saddle point $\left(x^{\star}, z^{\star}\right)$. More specifically, $\nabla_{x x} F(x, z) \succ 0$ and $\nabla_{z z} F(x, z) \prec 0$ for all $(x, z) \in U$.

The local strong or strict convexity-concavity assumption is very commonly used in literature for showing asymptotic convergence of saddle-point dynamics to the optimal solution of (26) (see, e.g., [5], [12]). Using this, the following result can be stated.

Lemma 9. Let Assumption 6 hold for some open neighborhood $U \subset \mathbb{R}^{n} \times \mathbb{R}^{m}$ of the saddle-point $\left(x^{\star}, z^{\star}\right)$. Then, the Hessian of $F$ given as

$$
\nabla^{2} F(x, z)=\left[\begin{array}{ll}
\nabla_{x x} F(x, z) & \nabla_{x z} F(x, z) \\
\nabla_{z x} F(x, z) & \nabla_{z z} F(x, z)
\end{array}\right],
$$

is invertible for all $(x, z) \in U$.

The proof is provided in Appendix D. Authors in [5] use the following saddle-point dynamics

$$
\dot{x}=-\nabla F_{x}(x, z), \quad \dot{z}=\nabla F_{z}(x, z) .
$$

and show asymptotic convergence to the saddle-point $\left(x^{\star}, z^{\star}\right)$ under Assumption 6. Next, the flow in (29) is modified so that fixed-time convergence can be guaranteed. The FxTS Newton's method is used to define the FxTS saddle-point (FxTS-SP) dynamics as

$$
\begin{aligned}
{\left[\begin{array}{c}
\dot{x} \\
\dot{z}
\end{array}\right]=} & -\left(\nabla^{2} F(x, z)\right)^{-1}\left(c_{1} \frac{\nabla F(x, z)}{\|\nabla F(x, z)\|^{\frac{p_{1}-2}{p_{1}-1}}}\right. \\
& \left.+c_{2} \frac{\nabla F(x, z)}{\|\nabla F(x, z)\|^{\frac{p_{2}-2}{p_{2}-1}}}\right),
\end{aligned}
$$

where $c_{1}, c_{2}>0, p_{1}>2,1<p_{2}<2$, and $\nabla F(x, z) \triangleq$ $\left[\begin{array}{ll}\nabla_{x} F(x, z)^{T} & \nabla_{z} F(x, z)^{T}\end{array}\right]^{T}$. Note that per Lemma 5, the point $(x, z)$ is an equilibrium point of (30) if and only if it satisfies $\nabla F(x, z)=0$. Using strict convexity-concavity of $F$ in $U$, one obtains that $\nabla F(x, z)=0$ implies $x=x^{\star}$ and $z=z^{\star}$. The first main result of this section is presented below.

Theorem 5. If F satisfies Assumption 6 for some $U \subset \mathbb{R}^{n} \times$ $\mathbb{R}^{m}$, then the trajectories of (30) converge to the saddle point $\left(x^{\star}, z^{\star}\right)$ in fixed time $T_{S P}$ for all $(x(0), z(0)) \in D \subset U$ 
where $D$ is the largest compact sub-level set of $V(x, z)=$ $\frac{1}{2}\|\nabla F(x, z)\|^{2}$ in $U$. Furthermore, if $U=\mathbb{R}^{n} \times \mathbb{R}^{m}$, then the results holds for all $(x(0), z(0)) \in \mathbb{R}^{n} \times \mathbb{R}^{m}$.

Proof: Consider the candidate Lyapunov function $V(x, z)=\frac{1}{2}\|\nabla F(x, z)\|^{2}$. Define $D$ as the largest compact sub-level set of $V$. Using analysis similar to the proof of Theorem 3, the time derivative of $V$ along the trajectories of (30) can be bounded as

$$
\dot{V} \leq-c_{1} 2^{\frac{\alpha_{1}}{2}} V^{\frac{\alpha_{1}}{2}}-c_{2} 2^{\frac{\alpha_{2}}{2}} V^{\frac{\alpha_{2}}{2}}
$$

where $\alpha_{1}=2-\frac{p_{1}-2}{p_{1}-1}$ and $\alpha_{2}=2-\frac{p_{2}-2}{p_{2}-1}$. It follows that for all $t \geq T_{S P}, \nabla F(x(t), z(t))=0$, or equivalently, $(x(t), z(t))=\left(x^{\star}, z^{\star}\right)$, where $T_{S P} \leq \frac{2^{1-\frac{\alpha_{1}}{2}}}{c_{1}\left(2-\alpha_{1}\right)}+\frac{2^{1-\frac{\alpha_{2}}{2}}}{c_{2}\left(\alpha_{2}-2\right)}$ for all $(x(0), z(0)) \in D$.

For the case when $U=\mathbb{R}^{n} \times \mathbb{R}^{m}$, the sub-level sets of $\|\nabla F\|$ are bounded and so, $V$ is radially unbounded. Therefore, the trajectories of (30) converge to the saddle-point of (26) for all $(x(0), z(0)) \in \mathbb{R}^{n} \times \mathbb{R}^{m}$.

Assumption 6 ensures that the Hessian $\nabla^{2} F(x, z)$ is invertible for all $(x, z) \in U$, and that the the saddle-point of $F$ is the only critical point. Per the analysis in Lemma 9, a sufficient condition for the Hessian to be invertible is that $\nabla_{x x} F$ is invertible and $\nabla_{z x} F$ is full row-rank. On the basis of this observation, the following result can be stated.

Corollary 1. Suppose there exists an open set $U \subset \mathbb{R}^{n} \times$ $\mathbb{R}^{m}$ such that $\nabla_{x x} F(x, z)$ is invertible and $\nabla_{z x} F(x, z)$ is full row-rank for all $(x, z) \in U$. Then, the trajectories of (30) converge to the set of the critical points of $F$, defined as $\Omega_{U}=$ $\{(x, z) \in U \mid \nabla F(x, z)=0\}$ in a fixed time $T_{S P}$ for all $(x(0), z(0)) \in D \subset U$, where $D$ is the largest compact sublevel set of $V=\frac{1}{2}\|\nabla F\|^{2}$ in $U$.

Note that Corollary 1 does not require strict convexityconcavity of $F$. Also, if the set $\Omega_{U}$ contains only the saddlepoint, i.e., the only critical point of the function $F$ in $\Omega_{U}$ is the saddle-point, then Corollary 1 guarantees local convergence of trajectories of (30) to the saddle-point in fixed time. The final remark to connect the results in Corollary 1 with those in Section III-D are made below.

Remark 7. For problem (17) with strictly convex $f$ and full row-rank matrix $A$, the conditions of Corollary 1 are satisfied. Furthermore, it is known that for this problem, the Karush-Kuhn-Tucker (KKT) conditions are also sufficient for optimality, i.e., the critical point $(\bar{x}, \bar{z})$ such that $\nabla F(\bar{x}, \bar{z}) \triangleq$ $\left[\begin{array}{c}\nabla f(\bar{x})+A^{T} \bar{z} \\ A \bar{x}-b\end{array}\right]=0$ is also the optimal point, i.e., $\left(x^{\star}, z^{\star}\right)=$ $(\bar{x}, \bar{z})$. Hence, one can use (30) with $F(x, z)=L(x, z)$ for the problems of the form (17), in the case when the conjugate function $f^{*}$ is not known in the closed-form.

This is formally shown in the following result.

Corollary 2. Consider the optimization problem (17). Assume that Assumption 4 holds and that $f \in C^{2}\left(\mathbb{R}^{n}, \mathbb{R}\right)$ is strictly convex. Then, with $F(x, z)=f(x)+z^{T}(A x-b)$, the trajectories of (30) reach the saddle-point $\left(x^{\star}, z^{\star}\right)$, where $x^{\star}$ is the solution of (17), in fixed time $T_{\text {eq2 }}<\infty$ for all $(x(0), z(0)) \in D \subset U$, where $D$ is the largest compact sublevel set of $V=\frac{1}{2}\|\nabla F\|^{2}$ in $U$.

Proof: Define $F(x, z)=f(x)+z^{T}(A x-b)$. Note that for strictly convex $f, \nabla_{x x} F=\nabla^{2} f(x)$ is invertible. Furthermore, $\nabla_{z x} F=A$ is full row-rank, which implies that the conditions of Corollary 1 are satisfied. Hence, the trajectories of (30) reach the set of points $(x, z)$ such that $\nabla F(x, z)=0$. Hence, one obtains that the trajectories of (30) for $F(x, z)=f(x)+$ $z^{T}(A x-b)$ reach the optimal point of (17) in fixed time $T_{e q 2} \leq$ $\frac{2^{1-\frac{\alpha_{1}}{2}}}{c_{1}\left(2-\alpha_{1}\right)}+\frac{2^{1-\frac{\alpha_{2}}{2}}}{c_{2}\left(\alpha_{2}-2\right)}$.

In summary, (30) can be used to solve constrained optimization problems of the form (17) as well as min-max problems of the form (26), and the optimal solutions can be obtained within a fixed time. Compared to [5], [12], where asymptotic convergence is studied for min-max problems of the form (26), and for (17) posed as saddle-point problem, the proposed method guarantees convergence within a fixed time under relaxed assumptions.

The proposed method (30) requires computation of the inverse of the Hessian matrix $\nabla^{2} F(x, z)$, which can be computationally expensive for problems with large $n, m$. Next, a first-order e, i.e., a method only requiring the gradient of the function $F$, is proposed under a stronger assumption on the function $F$.

Assumption 7. A saddle point $\left(x^{\star}, z^{\star}\right)$ exists that solves (26) and $F \in C^{2}\left(\mathbb{R}^{n} \times \mathbb{R}^{m}, \mathbb{R}\right)$ is locally strongly convex-concave on open neighborhood $U \subset \mathbb{R}^{n} \times \mathbb{R}^{m}$ of the saddle point $\left(x^{\star}, z^{\star}\right)$, i.e., there exist $k_{x}, k_{z}>0$ such that $\nabla_{x x} F(x, z) \succeq$ $k_{x} I$ and $\nabla_{z z} F(x, z) \preceq-k_{z} I$ for all $(x, z) \in U$.

Consider the following FxTS-GF based modified saddlepoint dynamics

$$
\left[\begin{array}{c}
\dot{x} \\
\dot{z}
\end{array}\right]=-c_{1} \frac{\tilde{\nabla} F(x, z)}{\|\nabla F(x, z)\|^{\frac{p_{1}-2}{p_{1}-1}}}-c_{2} \frac{\tilde{\nabla} F(x, z)}{\|\nabla F(x, z)\|^{\frac{p_{2}-2}{p_{2}-1}}},
$$

where $c_{1}, c_{2}>0, p_{1}>2,1<p_{2}<2, \tilde{\nabla} F(x, z) \triangleq$ $\left[\nabla_{x} F(x, z)^{T}-\nabla_{z} F(x, z)^{T}\right]^{T}$. Note that (29) is a special case of (29) with $c_{1}=1, c_{2}=0$ and $p_{1}=2$. The following result can be readily stated for (29).

Theorem 6. Suppose the function F satisfies Assumption 7. Then, the trajectories of (29) converge to the saddle-point in a fixed time $T_{S P 2}$ for all $(x(0), z(0)) \in U$. If $U=\mathbb{R}^{n} \times \mathbb{R}^{n}$, then the result holds for all $(x(0), z(0)) \in \mathbb{R}^{n} \times \mathbb{R}^{m}$.

Proof: Choose the candidate Lyapunov function as $V(x, z)=\frac{1}{2}\|\nabla F(x, z)\|^{2}$. The time derivative of $V$ along the trajectories of (29) reads as in (30). Now, using the strong convexity-concavity of $F$, one obtains

$$
\begin{aligned}
\dot{V} & \leq-c_{1} k_{x} \frac{\left\|\nabla_{x} F\right\|^{2}}{\|\nabla F\|^{\frac{p_{1}-2}{p_{1}-1}}}-c_{2} k_{x} \frac{\left\|\nabla_{x} F\right\|^{2}}{\|\nabla F\|^{\frac{p_{1}-2}{p_{1}-1}}} \\
& -c_{1} k_{z} \frac{\left\|\nabla_{z} F\right\|^{2}}{\|\nabla F\|^{\frac{p_{1}-2}{p_{1}-1}}}-c_{2} k_{z} \frac{\left\|\nabla_{z} F\right\|^{2}}{\|\nabla F\|^{\frac{p_{1}-2}{p_{1}-1}}} \\
& \leq-c_{1} k \frac{\|\nabla F\|^{2}}{\|\nabla F\|^{\frac{p_{1}-2}{p_{1}-1}}}-c_{2} k \frac{\|\nabla F\|^{2}}{\|\nabla F\|^{\frac{p_{1}-2}{p_{1}-1}}}=-k_{7} V^{\frac{\alpha_{7}}{2}}-k_{8} V^{\frac{\alpha_{8}}{2}},
\end{aligned}
$$




$$
\begin{aligned}
\dot{V} & =\nabla_{x} F^{T} \nabla_{x x} F \dot{x}+\nabla_{x} F^{T} \nabla_{x z} F \dot{z}+\nabla_{z} F^{T} \nabla_{z x} F \dot{x}+\nabla_{z} F^{T} \nabla_{z z} F \dot{z} \stackrel{(29)}{=} \nabla_{x} F^{T} \nabla_{x x} F \dot{x}+\nabla_{z} F^{T} \nabla_{z z} F \dot{z} \\
& =-\nabla_{x} F^{T} \nabla_{x x} F\left(c_{1} \frac{\nabla F_{x}(x, z)}{\|\nabla F(x, z)\|^{\frac{p_{1}-2}{p_{1}-1}}}+c_{2} \frac{\nabla F_{x}(x, z)}{\|\nabla F(x, z)\|^{\frac{p_{2}-2}{p_{2}-1}}}\right)+\nabla_{z} F^{T} \nabla_{z z} F\left(c_{1} \frac{\nabla F_{z}(x, z)}{\| \nabla F(x, z)^{\frac{p_{1}-2}{p_{1}-1}}}+c_{2} \frac{\nabla F_{z}(x, z)}{\|\nabla F(x, z)\|^{\frac{p_{2}-2}{p_{2}-1}}}\right) .
\end{aligned}
$$

where $k_{7}=c_{1} k 2^{\frac{\alpha_{7}}{2}}, k_{8}=c_{2} k 2^{\frac{\alpha_{8}}{2}}, 0<\alpha_{7}=2-\frac{p_{1}-2}{p_{1}-1}<2$ and $\alpha_{8}=2-\frac{p_{2}-2}{p_{2}-1}>2$, where $k=\min \left\{k_{x}, k_{z}\right\}$. Hence, using Lemma 2, one obtains that the optimal point of (26) can be found in fixed time $T_{S P 2}$ satisfying $T_{S P 2} \leq \frac{2}{k_{7}\left(2-\alpha_{7}\right)}+$ $\frac{2}{k_{8}\left(\alpha_{8}-2\right)}$. Furthermore, the norm of the gradient $\|\nabla F\|$ is radially unbounded on $U$, and hence, for $U=\mathbb{R}^{n} \times \mathbb{R}^{m}$, the result holds globally for any $(x(0), z(0))$.

\section{NUMERICAL EXAMPLES}

The efficacy of the proposed methods is illustrated via three numerical examples. The computations are done using MATLAB R2018a on a desktop with a 32GB DDR3 RAM and an Intel Xeon E3-1245 processor (3.4 GHz). Unless mentioned otherwise, Euler discretization is used for MATLAB implementation with time-step $d t=10^{-5}$, and with constant step-size, the convergence time $T$ in seconds translates to $T \times 10^{5}$ iterations. In the first example, an instance of the logistic regression based support-vector machine is considered, where the performance of the proposed FxTS-GF is compared with Newton's method. The flow in (8) is used to find the optimal solution, i.e., the separating hyperplane for a given labelled data set, within a fixed time. In the second example, an instance of QP with equality constraints is considered as a constrained convex optimization problem (17). The FxTS saddle-point dynamics in (30) is used to find the optimal point of the problem, and to illustrate that for any initial condition, the optimal point can be found within a fixed time. Finally, an instance of the min-max problem (26) is considered, and the FxTS saddle-point dynamics in (30) is used to find the saddle-point.

\section{A. Support Vector Machine: Unconstrained optimization}

Consider an instance of logistic SVM, where the function $f: \mathbb{R}^{2} \rightarrow \mathbb{R}$ is given as

$$
f(x)=\frac{1}{2}\|x\|^{2}+\frac{1}{\mu} \sum_{i=1}^{N} \log \left(1+\exp \left(-\mu l_{i} x^{T} z_{i}\right)\right),
$$

with $\mu>0$ a large positive number. Here $x=x^{\star} \in \mathbb{R}^{2}$ represents the separating hyperplane, and $z_{i} \in \mathbb{R}^{2}$ and $l_{i} \in\{-1,1\}$ denote the $i$-th data point and its corresponding label, respectively. The vectors $z_{i}$ are chosen from a random distribution around the line $x_{1}=x_{2}$, so that the solution $x^{\star}$, i.e., the separating hyperplane, to the minimization problem $\min _{x \in \mathbb{R}^{n}} f(x)$ is the vector $[1,-1]$. In this case, $N=500$ randomly distributed data points are considered. The parameters used in the numerical simulations are $c_{1}=c_{2}=10$, $p_{1}=2.6, p_{2}=1.6$, and $\mu=2$. The theoretical bound on

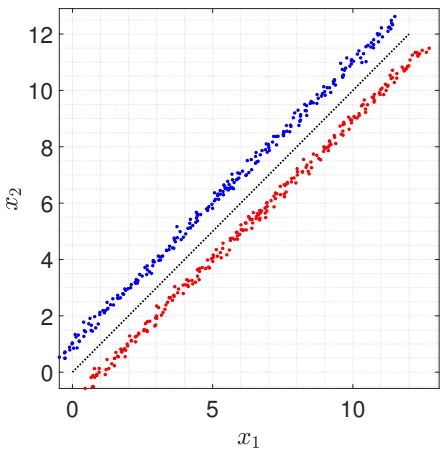

Fig. 1. Distribution of points $z_{i}$ around the line $x_{1}=x_{2}$ (red dotted line). Blue and red stars denote the points corresponding to $l_{i}=-1$ and $l_{i}=1$, respectively. .

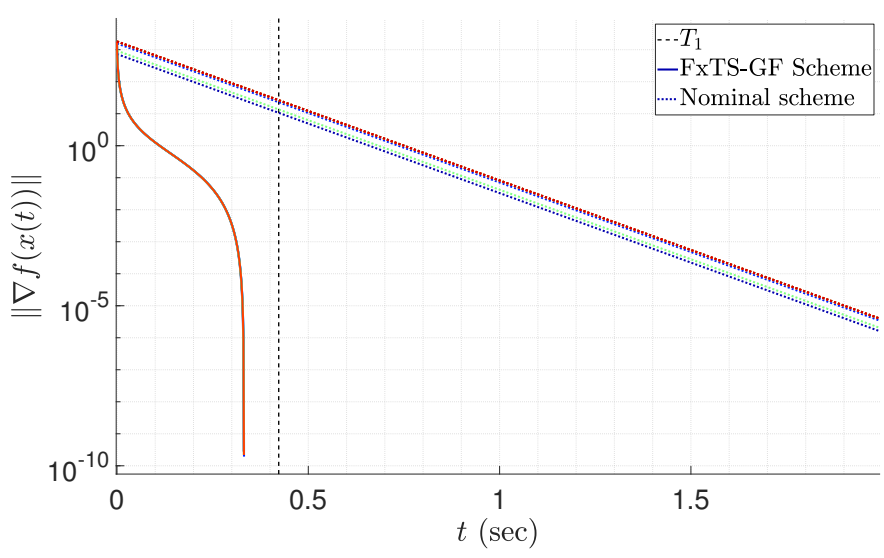

Fig. 2. The norm of gradient, $\|\nabla f(x(t))\|$, with time for various initial conditions for the FxTS-GF (solid lines) and the Newton's method (dotted lines).

the time of convergence is $T_{1} \leq 0.43$. Figure 1 shows the distribution of $z_{i}$ around the line $x_{1}=x_{2}$.

Figure 2 depicts the norm of the gradient $\|\nabla f(x(t))\|$ with time for various initial conditions. The log scale is used on $y-$ axis so that the variation of the norm $\|\nabla f\|$ is clearly shown for values near zero, and the super-linear nature of convergence can be demonstrated. Note that the plots corresponding to the nominal GF method are linear on the log-scale, which verifies that the convergence is exponential, while the curved plots of the proposed scheme illustrate the super-linear convergence. It can also be noted that the convergence time (upto the error of $10^{-10}$ ) is bounded by the theoretical bound of $T_{1}$. Thus, per Figure 2, nominal GF takes at least 5 times more iterations as compared to the FxTS-GF, in order to converge to the same level of accuracy. 


\section{B. Example 2: $Q P$ with equality constraints}

Consider (25) with $x \in \mathbb{R}^{10}$ and $A \in \mathbb{R}^{5 \times 10}$. For simplicity, consider a diagonal matrix $Q$ with positive diagonal elements and a full row-rank matrix $A$, so that all the conditions of Corollary 2 are satisfied. The values of $Q, A, b, c$ are chosen through random matrix generator in MATLAB. The following parameters are used for FxTS-SP dynamics in (30): $c_{1}=10, c_{2}=10, p_{1}=2.2, p_{2}=1.8$. With these parameters, the upper bound on the time of convergence in Corollary 2 satisfies $T_{e q 2}=T_{S P} \leq 1.0025$.

Figure 3 compares the performance of the proposed method relative to Newton's method for saddle-point dynamics, i.e., (30) with $c_{2}=0$ and $p_{2}=2$. The dotted lines illustrate the evolution of Newton's method, while solid lines illustrate that of FxTS-SP dynamics (30). The vertical black dashed black line corresponds to $T_{S P}=1.0025 \mathrm{sec}$. Figure 3 shows the variation of $\left\|x(t)-x^{\star}\right\|$ with time for various initial conditions. The proposed scheme converges to the error of magnitude less than $10^{-8}$ within $T_{S P}$ sec, while the nominal scheme takes longer time (and thus, more number of iterations) to achieve the same. It can also be seen that the convergence time is always bounded by $T_{S P}$ for all initial conditions for the proposed method.

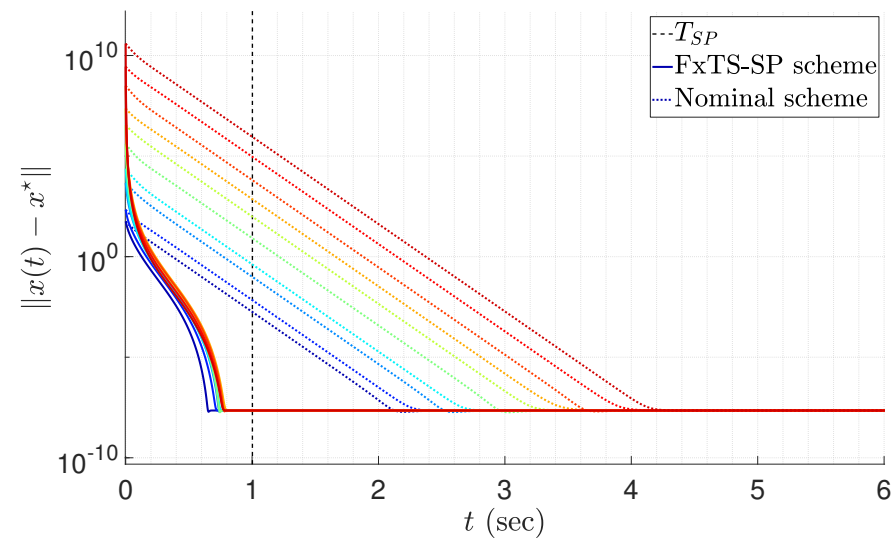

Fig. 3. The norm $\left\|x(t)-x^{\star}\right\|$ with time for various initial conditions for nominal saddle-point dynamics $\left(p_{1}=2, c_{2}=0\right)$ and FxTS saddle-point dynamics $\left(p_{1}=2.2, p_{2}=1.8\right)$.

\section{Example 3: Min-max problem}

A numerical example for the min-max problem $\max \min F(x, z)$ is considered, where the function $F$ is defined as:

$$
F(x, z)=(\|x\|-1)^{4}-\|z\|^{2}\|x\|^{2},
$$

with $x \in \mathbb{R}^{n}$ and $z \in \mathbb{R}^{m}$. The dimensions are chosen as $n=3$ and $m=1$. The set of optimal points $(x, z)$ satisfy $\|x\|=1,\|z\|=0$ [5], i.e., the optimal point is not unique in this case. The parameters $c_{1}, c_{2}$ are chosen as $c_{1}=c_{2}=10$.

The first case study considers a varying range of initial conditions $(x(0), z(0))$ and constant values of the parameters $p_{1}, p_{2}$, chosen as $p_{1}=2.2$ and $p_{2}=1.8$. Figure 4 shows the convergence time (upto an error of $\|\nabla F(x, z)\| \leq 10^{-15}$ ) for various initial conditions $x(0), z(0)$. The results illustrate that the time of convergence does not depend upon the initial distance from the saddle point. Also, the actual time of convergence $T_{c}$ is lower than the upper bound $T_{S P}$.

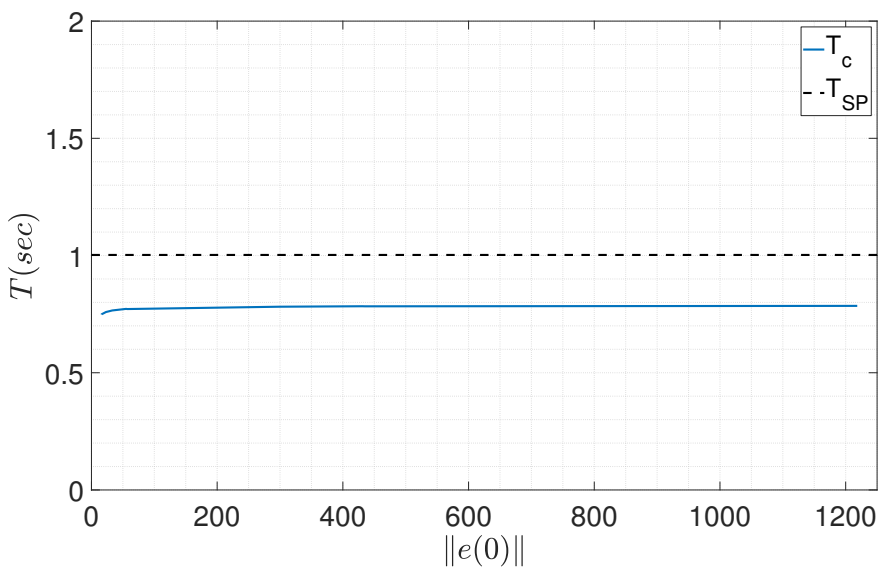

Fig. 4. Time of convergence $T_{c}$ with norm of the initial error $\|e(0)\| \triangleq$ $\left\|\left[\left(x(0)-x^{\star}\right)^{T}\left(z(0)-z^{\star}\right)^{T}\right]^{T}\right\|$.

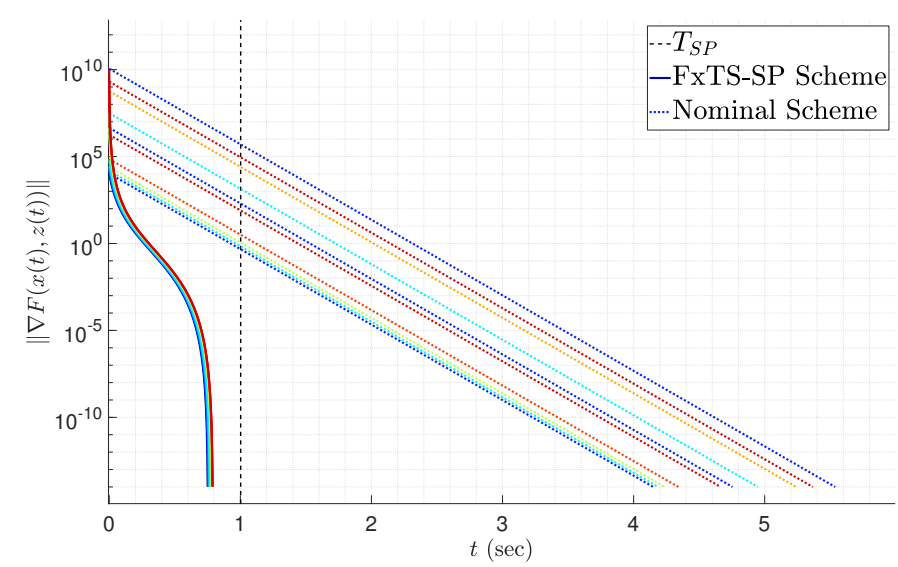

Fig. 5. The norm of the gradient, $\|\nabla F(x(t), z(t))\|$, with time for various initial conditions for nominal saddle-point dynamics $\left(p_{1}=2, c_{2}=0\right)$ and FxTS saddle-point dynamics $\left(p_{1}=2.2, p_{2}=1.8\right)$.

Figure 5 illustrates the convergence of norm of the gradient, $\|\nabla F(x, z)\|$, to zero in fixed time for various initial conditions. Figure 6 and 7 plot the norm of the error $x-x^{\star}$ and $z-z^{\star}$, respectively, for various initial conditions. Solid lines show the performance of the proposed method (30), and dotted lines show the performance of Newton's method $\left(c_{2}=0, p_{2}=2\right)$ when solving for saddle-point dynamics. Again, it can be noted from the figures that the proposed method converges within the theoretical bound on $T_{S P}$, and has super-linear convergence rate.

The second case study considers that the parameters $p_{1}, p_{2}$ are varied in the ranges $[2,2.2]$ and $[1.8,2]$, respectively. Figure 8 shows the norm of the gradient, $\|\nabla F(x, z)\|$, with time. As can be seen in the Figure 8, the case when $p_{1}=$ $p_{2}=2$ has linear convergence (straight line on the log plot), while for $p_{1}>2$ and $p_{2}<2$, the convergence is super-linear. It can also be observed that as $p_{1}$ increases and $p_{2}$ decreases, the convergence becomes faster and the time of convergence becomes smaller. 


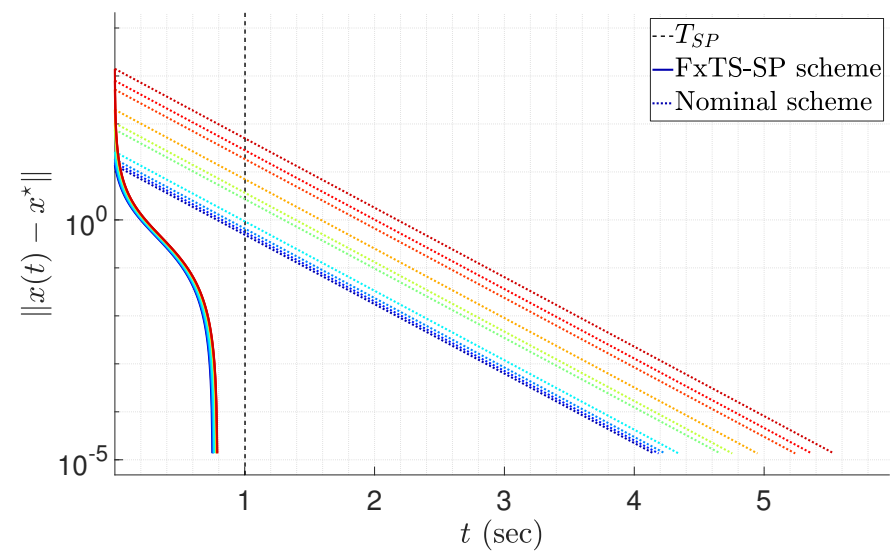

Fig. 6. The norm $\left\|x-x^{\star}\right\|$ with time for various initial conditions for nominal saddle-point dynamics $\left(p_{1}=2, c_{2}=0\right)$ and FxTS saddle-point dynamics $\left(p_{1}=2.2, p_{2}=1.8\right)$.

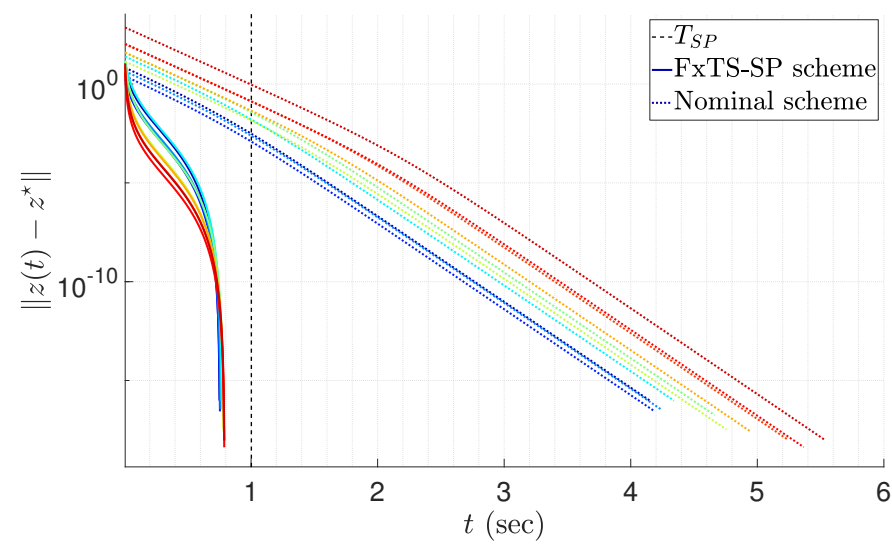

Fig. 7. The norm $\left\|z-z^{\star}\right\|$ with time for various initial conditions for nominal saddle-point dynamics $\left(p_{1}=2, c_{2}=0\right)$ and FxTS saddle-point dynamics $\left(p_{1}=2.2, p_{2}=1.8\right)$.

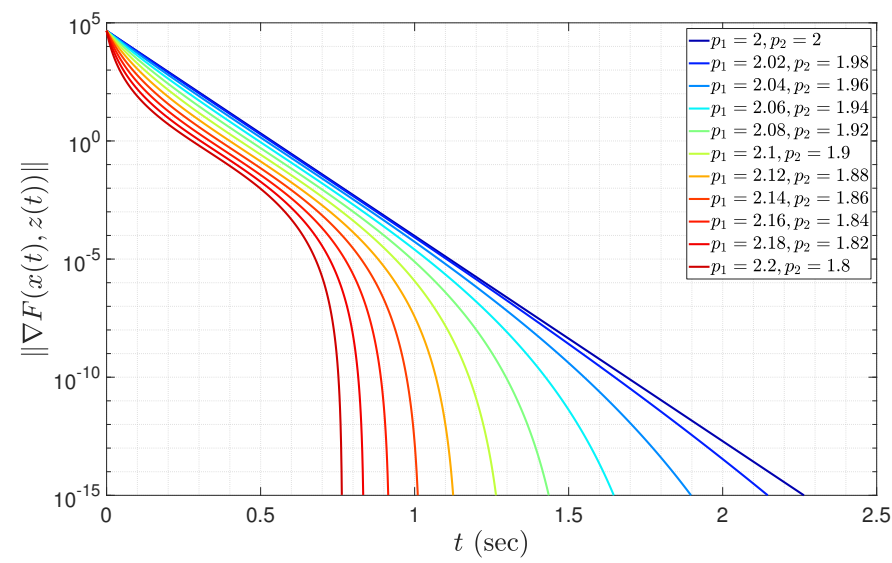

Fig. 8. The norm of the gradient, $\|\nabla F(x(t), z(t))\|$, with time for various $p_{1}, p_{2}$.

The implementation of the proposed method in numerical studies is done using Euler integration with constant step size. Figure 9 shows the performance of the proposed method for various values of discretization steps between $10^{-2}$ and $10^{-6}$. As the figure suggests, the discretization step does not affect the convergence performance of the proposed method.

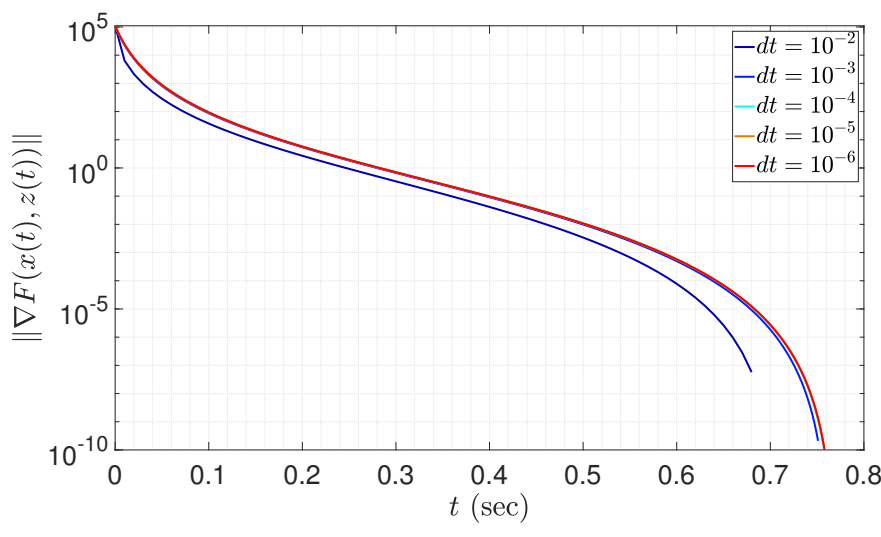

Fig. 9. The norm of the gradient, $\|\nabla F(x(t), z(t))\|$, with time for various $p_{1}, p_{2}$.

Finally, the performance of the proposed method is compared with the performance of the rescaled gradient flow (6). More specifically, the considered rescaled-gradient flow scheme is

$$
\left[\begin{array}{c}
\dot{x} \\
\dot{z}
\end{array}\right]=-c_{1}\left(\nabla^{2} F(x, z)\right)^{-1} \frac{\nabla F(x, z)}{\|\nabla F(x, z)\|^{\frac{p_{1}-2}{p_{1}-1}}} .
$$

where $0<\theta<1$. Since the objective function in (31) is only strictly convex-concave and not strongly convex-concave, (29) cannot be used, but (30) can be used. The dynamical system (32) is a Newton's modification of rescaled-gradient flow (6) discussed in [3], where Hessian is used so that (32) can be used for a strictly convex-concave function.

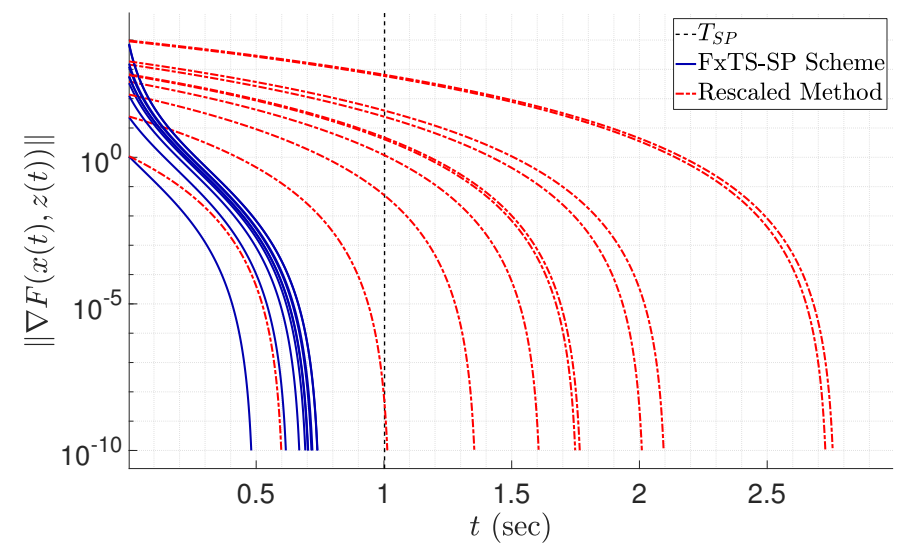

Fig. 10. The norm of gradient, $\|\nabla F(x(t), z(t))\|$, with time for various initial conditions for the proposed scheme and the rescaled gradient flow scheme.

Figure 10 plots the norm of the gradient for various initial conditions, where $p_{1}=2.2, p_{2}=1.8, c_{1}=c_{2}=10$ for (30), $p_{1}=2.2, c_{1}=10$ for (32). It can be seen that the convergence of the rescaled gradient flow scheme (32) is super-linear (finite-time convergence), but slower than the proposed scheme. It is evident from Figure 10 that the time of convergence for (32) grows as $\left\|x(0)-x^{\star}\right\|$ increases, while that of the proposed scheme (30) remains bounded.

Figure 11 depicts the wall-clock time (i.e., actual run-time) for the two aforementioned schemes. The results are presented for 1000 trials, where the simulations are run until the norm 


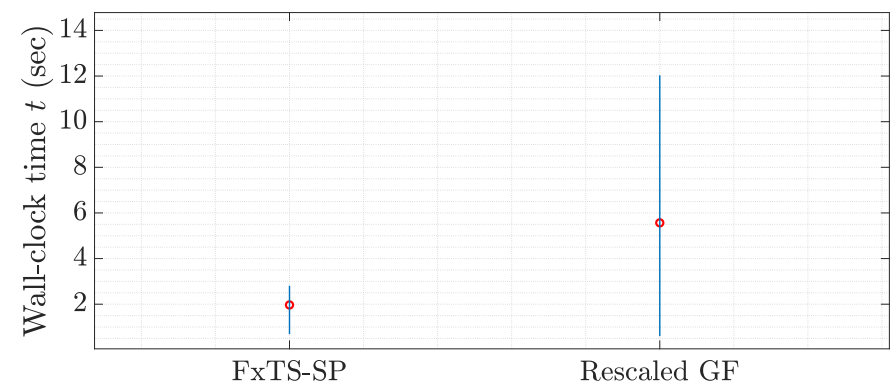

Fig. 11. The wall-clock time for 1000 trials for the proposed scheme, rescaled gradient flow based scheme. The red dot represents the mean value for the 1000 trials while the vertical lines represent the minimum and maximum values of the respective schemes.

of the gradient, $\|\nabla F(x(t), z(t))\|$, drops below $10^{-10}$. It is clear from the figures the proposed scheme takes smaller computation time than the accelerated scheme, while giving better convergence rate. Note that the wall-clock time, which corresponds to the actual computational time, is different from the convergence time $T_{S P}$, which, in the discrete setting, corresponds to the number of steps required for the convergence per the relation $N=T_{S P} \times 10^{5}$. It is evident that the proposed method performs better than the nominal Newton's method, as well as some of the very commonly used accelerated methods, both in terms of number of iterations required for converging to a small neighborhood of the optimal solution, and wall-clock time. In the next section, we discuss some of the limitations of the study of continuous-time optimization theory, and lay out directions for future work, based on recent developments in the field of rate-preserving and consistent discretization schemes.

\section{DISCUSSION}

While optimization methods in continuous-time are important and have major theoretical relevance in general, only discrete-time algorithms are of practical use. It is an open question as to how one can discretize the dynamics (8) and other schemes presented in this work, so that the fixedtime convergence guarantees are provably preserved. While in all the numerical examples the performance of discretized implementation is at par with the theoretical results, i.e., the convergence is super-linear and the time of convergence is upper bounded by the theoretically established upper bound, the theoretical investigation on how the convergence properties are preserved after discretization is an open problem, and an active field of research (see [34], [35]).

In [34], the authors study a particular class of homogeneous systems, and show that there exists a consistent discretization scheme that preserves the finite-time convergence. They extend their results to practically FxTS systems in [35], where they show that the trajectories of the discretized system reach to an arbitrary small neighborhood of the equilibrium point in fixed time, independent of the initial condition. Given that the provided numerical examples suggest that the proposed method works efficiently even with constant-step Euler integration, the questions that naturally arise are: (i) how could the theory of consistent discretization be extended to a more general class of FTS and FxTS systems, and (ii) how this theory could be used for the methods developed in this paper so that exact convergence of iterative discrete-time optimization schemes for the proposed methods can be guaranteed in a finite or fixed number of steps. These topics are beyond the scope of the current paper, and are left open for future research.

\section{COnCLusions And Future Work}

This paper presented modified GF schemes that provide convergence of the solution to the optimal point in fixed time, under various assumptions such as strict convexity and gradient dominance, which is a relaxation of strong-convexity. A modified version of Newton's method is also presented that possesses fixed-time convergence guarantees from any given initial condition for optimization problems with strictly convex objective function. Based on this result, a novel method is proposed to find the optimal point of a convex optimization problem with linear equality constraints in fixed time. A modified scheme for the saddle-point dynamics is proposed so that the min-max problem can be solved in fixed time. Though all the methods are presented for continuous-time optimization, numerical examples illustrate that the proposed schemes have super-linear convergence in the discretized implementation as well, that the time of convergence satisfies the theoretical bound, and that the performance of the proposed method is better than the one of commonly used algorithms, such as Newton's method, the rescaled gradient-based method.

Studying the general optimization problem with both equality and inequality constraints is part of the future investigations, where schemes that can converge to the optimal point in fixed time will be designed. Also, it will be of great interest to study FTS and FxTS methods of optimization on function spaces with applications such as finding the optimal barrier function for control synthesis under spatio-temporal specifications and input constraints. Finally, as mentioned in Section VI, one of the future research directions is to investigate discretization schemes for FTS and FxTS systems that can preserve the time of convergence, and translate FTS and FxTS to convergence in finite and fixed number of steps, respectively.

\section{ACKNOWLEDGEMENTS}

The authors acknowledge Dr. Rohit Gupta for several fruitful discussions.

\section{REFERENCES}

[1] A. Brown and M. C. Bartholomew-Biggs, "Some effective methods for unconstrained optimization based on the solution of systems of ordinary differential equations," Journal of Optimization Theory and Applications, vol. 62, no. 2, pp. 211-224, 1989.

[2] A. Wibisono and A. C. Wilson, "On accelerated methods in optimization," arXiv preprint arXiv:1509.03616, 2015.

[3] A. Wibisono, A. C. Wilson, and M. I. Jordan, "A variational perspective on accelerated methods in optimization," Proceedings of the National Academy of Sciences, vol. 113, no. 47, pp. E7351-E7358, 2016.

[4] W. Su, S. Boyd, and E. Candes, "A differential equation for modeling Nesterov's accelerated gradient method: Theory and insights," in Advances in Neural Information Processing Systems, 2014, pp. 25102518 . 
[5] A. Cherukuri, B. Gharesifard, and J. Cortes, "Saddle-point dynamics: Conditions for asymptotic stability of saddle points," SIAM Journal on Control and Optimization, vol. 55, no. 1, pp. 486-511, 2017.

[6] N. K. Dhingra, S. Z. Khong, and M. R. Jovanović, "The proximal augmented lagrangian method for nonsmooth composite optimization," IEEE Transactions on Automatic Control, vol. 64, no. 7, pp. 2861-2868, 2018.

[7] S. Osher, F. Ruan, J. Xiong, Y. Yao, and W. Yin, "Sparse recovery via differential inclusions," Applied and Computational Harmonic Analysis, vol. 41, no. 2, pp. 436-469, 2016.

[8] H.-B. Dürr and C. Ebenbauer, "On a class of smooth optimization algorithms with applications in control," IFAC Proceedings Volumes, vol. 45, no. 17, pp. 291-298, 2012.

[9] W. Krichene, A. Bayen, and P. L. Bartlett, "Accelerated mirror descent in continuous and discrete time," in Advances in Neural Information Processing Systems, 2015, pp. 2845-2853.

[10] U. Helmke and J. B. Moore, Optimization and Dynamical Systems. Springer Science \& Business Media, 2012.

[11] J. Schropp and I. Singer, "A dynamical systems approach to constrained minimization," Numerical Functional Analysis and Optimization, vol. 21, no. 3-4, pp. 537-551, 2000.

[12] A. Cherukuri, E. Mallada, S. Low, and J. Cortés, "The role of convexity on saddle-point dynamics: Lyapunov function and robustness," IEEE Transactions on Automatic Control, 2017.

[13] U. Clarenz, S. Henn, M. Rumpf, and K. Witsch, "Relations between optimization and gradient flow methods with applications to image registration," in Proceedings of the 18th GAMM-Seminar Leipzig on Multigrid and Related Methods for Optimization Problems, 2002.

[14] J. Cortés, "Finite-time convergent gradient flows with applications to network consensus," Automatica, vol. 42, no. 11, pp. 1993-2000, 2006.

[15] S. Boyd and L. Vandenberghe, Convex Optimization. Cambridge University Press, 2004.

[16] H. Karimi, J. Nutini, and M. Schmidt, "Linear convergence of gradient and proximal-gradient methods under the Polyak- łojasiewicz condition," in Joint European Conference on Machine Learning and Knowledge Discovery in Databases. Springer, 2016, pp. 795-811.

[17] Y. Nesterov, "Accelerating the cubic regularization of Newton's method on convex problems," Mathematical Programming, vol. 112, no. 1, pp. 159-181, 2008.

[18] J. Wang and N. Elia, "A control perspective for centralized and distributed convex optimization," in IEEE Conference on Decision and Control and European Control Conference. IEEE, 2011, pp. 3800 3805 .

[19] X. Ma and N. Elia, "A distributed continuous-time gradient dynamics approach for the active power loss minimizations," in 2013 51st Annual Allerton Conference on Communication, Control, and Computing. IEEE, 2013, pp. 100-106.

[20] D. Feijer and F. Paganini, "Stability of primal-dual gradient dynamics and applications to network optimization," Automatica, vol. 46, no. 12, pp. 1974-1981, 2010.

[21] B. Gharesifard and J. Cortés, "Distributed convergence to nash equilibria in two-network zero-sum games," Automatica, vol. 49, no. 6, pp. 1683 $1692,2013$.

[22] M. Benzi, G. H. Golub, and J. Liesen, "Numerical solution of saddle point problems," Acta numerica, vol. 14, pp. 1-137, 2005.

[23] J. C. Duchi, M. I. Jordan, and M. J. Wainwright, "Minimax optimal procedures for locally private estimation," Journal of the American Statistical Association, vol. 113, no. 521, pp. 182-201, 2018.

[24] G. Qu and N. Li, "On the exponential stability of primal-dual gradient dynamics," IEEE Control Systems Letters, vol. 3, no. 1, pp. 43-48, 2019.

[25] S. P. Bhat and D. S. Bernstein, "Finite-time stability of continuous autonomous systems," SIAM Journal of Control and Optimization, vol. 38, no. 3, pp. 751-766, 2000.

[26] A. Polyakov, "Nonlinear feedback design for fixed-time stabilization of linear control systems," IEEE Transactions on Automatic Control, vol. 57, no. 8, pp. 2106-2110, 2012.

[27] F. Chen and W. Ren, "Convex optimization via finite-time projected gradient flows," in IEEE Conference on Decision and Control. IEEE, 2018, pp. 4072-4077.

[28] Y. Song and W. Chen, "Finite-time convergent distributed consensus optimisation over networks," IET Control Theory \& Applications, vol. 10, no. 11, pp. 1314-1318, 2016.

[29] X. Pan, Z. Liu, and Z. Chen, "Distributed optimization with finite-time convergence via discontinuous dynamics," in 2018 37th Chinese Control Conference. IEEE, 2018, pp. 6665-6669.
[30] C. Li, X. Yu, X. Zhou, and W. Ren, "A fixed time distributed optimization: A sliding mode perspective," in 43rd Annual Conference of the Industrial Electronics Society. IEEE, 2017, pp. 8201-8207.

[31] J. D. Sánchez-Torres, M. J. Loza-Lopez, R. Ruiz-Cruz, E. N. Sanchez, and A. G. Loukianov, "A fixed time convergent dynamical system to solve linear programming," in IEEE 53rd Annual Conference on Decision and Control. IEEE, 2014, pp. 5837-5842.

[32] Z. Feng and G. Hu, "Finite-time distributed optimization with quadratic objective functions under uncertain information," in 2017 IEEE 56th Conference on Decision and Control. IEEE, 2017, pp. 208-213.

[33] M. Santilli, A. Marino, and A. Gasparri, "A finite-time protocol for distributed continuous-time optimization of sum of locally coupled strictly convex functions," in IEEE 57th Conference on Decision and Control. IEEE, 2018, pp. 993-998.

[34] A. Polyakov, D. Efimov, and B. Brogliato, "Consistent discretization of finite-time stable homogeneous systems," in VSS 2018-15th International Workshop on Variable Structure Systems and Sliding Mode Control, 2018.

[35] — "Consistent discretization of finite-time and fixed-time stable systems," SIAM Journal on Control and Optimization, vol. 57, no. 1, pp. 78-103, 2019.

[36] A. Beck, Introduction to nonlinear optimization: Theory, algorithms, and applications with MATLAB. SIAM, 2014, vol. 19.

[37] K. Garg, M. Baranwal, R. Gupta, R. Vasudevan, and D. Panagou, "Fixedtime stable proximal dynamical system for solving mixed variational inequality problems," arXiv preprint arXiv:1908.03517, 2019.

[38] R. P. Agarwal and V. Lakshmikantham, Uniqueness and Nonuniqueness Criteria for Ordinary Differential Equations. World Scientific Publishing Company, 1993, vol. 6.

[39] R. T. Rockafellar, Convex Analysis. Princeton university press, 2015.

[40] I. M. Bomze and L. Palagi, "Quartic formulation of standard quadratic optimization problems," Journal of Global Optimization, vol. 32, no. 2, pp. 181-205, 2005.

[41] C. Zalinescu, Convex Analysis in General Vector Spaces. World Scientific, 2002.

[42] C. Paige, G. P. Styan, B.-Y. Wang, and F. Zhang, "Hua's matrix equality and schur complements," International Journal of Information \& Systems Sciences, vol. 4, no. 1, p. 124, 2008.

\section{APPENDiX A \\ PROOF OF LEMMA 5} only if

Proof: One has that $x=\bar{x}$ is an equilibrium of (8) if and

$$
\begin{aligned}
\dot{\bar{x}}=0 & \Longleftrightarrow-c_{1} \frac{\nabla f(\bar{x})}{\|\nabla f(\bar{x})\|^{\frac{p_{1}-2}{p_{1}-1}}}-c_{2} \frac{\nabla f(\bar{x})}{\|\nabla f(\bar{x})\|^{\frac{p_{2}-2}{p_{2}-1}}}=0 \\
& \Longleftrightarrow c_{1} \frac{\|\nabla f(\bar{x})\|}{\|\nabla f(\bar{x})\|^{\frac{p_{1}-2}{p_{1}-1}}}+c_{2} \frac{\|\nabla f(\bar{x})\|}{\|\nabla f(\bar{x})\|^{\frac{p_{2}-2}{p_{2}-1}}}=0 \\
& \Longleftrightarrow c_{1}\|\nabla f(\bar{x})\|^{1-\frac{p_{1}-2}{p_{1}-1}}+c_{2}\|\nabla f(\bar{x})\|^{1-\frac{p_{2}-2}{p_{2}-1}}=0, \\
& \Longleftrightarrow\|\nabla f(\bar{x})\|=0,
\end{aligned}
$$

since $1-\frac{p_{1}-2}{p_{1}-1}, 1-\frac{p_{2}-2}{p_{2}-1}>0$ for $p_{1}>2$ and $1<p_{2}<2$. Hence, $x=\bar{x}$ is an equilibrium point if and only if $\nabla f(\bar{x})=$ 0 . This completes the proof.

\section{APPENDiX B \\ PRoOf of LEMMA 6}

Proof: Let $\mathcal{X}=\{x \mid \nabla f(x)=0\}$. Since $f \in C_{\text {loc }}^{1,1}$, continuity of right-hand side of (8) is immediate on $\mathbb{R}^{n} \backslash \mathcal{X}$. Let $\bar{x} \in \mathcal{X}$ and $L$ be the Lipschitz constant for function $\nabla f$, i.e., $\|\nabla f(x)-\nabla f(y)\| \leq L\|x-y\|$ for $x, y \in D$ where $D$ is some open neighborhood of $\bar{x}$. For $y=\bar{x}$, it follows that $\|\nabla f(x)-\nabla f(\bar{x})\|=\|\nabla f(x)\| ;$ then, using continuity of the 
norm, one has

$$
\begin{aligned}
\left\|\lim _{x \rightarrow \bar{x}} c_{1} \frac{\nabla f(x)}{\|\nabla f(x)\|^{\frac{p_{1}-2}{p_{1}-1}}}\right\| & =\lim _{x \rightarrow \bar{x}} c_{1}\left\|\frac{\nabla f(x)}{\|\nabla f(x)\|^{\frac{p_{1}-2}{p_{1}-1}}}\right\| \\
& =c_{1} \lim _{x \rightarrow \bar{x}}\|\nabla f(x)\|^{1-\frac{p_{1}-2}{p_{1}-1}} \\
& =c_{1} \lim _{x \rightarrow \bar{x}}\|\nabla f(x)\|^{\delta_{1}} \\
& \leq c_{1} L^{\delta_{1}} \lim _{x \rightarrow \bar{x}}\|x-\bar{x}\|^{\delta_{1}}=0,
\end{aligned}
$$

where $\delta_{1}=1-\frac{p_{1}-2}{p_{1}-1}>0$ for $p_{1}>2$. Hence, one has that $\lim _{x \rightarrow \bar{x}} c_{1} \frac{\nabla f(x)}{\|\nabla f(x)\|^{\frac{p_{1}-2}{p_{1}-1}}}=0$. Similarly, it can be shown that $\lim _{x \rightarrow \bar{x}} c_{2} \frac{\nabla f}{\|\nabla f\|_{p_{2}-2}}=0$, since $\delta_{2}=1-\frac{p_{2}-2}{p_{2}-1}>0$ for all $1<p_{2}<2$. Per Lemma 5, one has that $\bar{x}$ is an equilibrium of (8). This implies that the right-hand side of (8) is continuous at $x=\bar{x}$, for all $\bar{x} \in \mathcal{X}$, and hence, is continuous for all $x \in \mathbb{R}^{n}$.

\section{APPENDIX C \\ PROOF OF LEMMA 7}

Proof: The convexity and strong-smoothness assumptions on $f$ implies that $f^{* *}=f$, i.e., $f$ is the conjugate of its conjugate $f^{*}$. Define $\kappa=f^{*}$ so that one has $\kappa^{*}=f^{* *}=$ $f$. Now, since the function $f$ is the conjugate of $\kappa$ and is $\beta$-strongly smooth, from [41, Section 3.5], one obtains that there exists $\beta^{*}$ such that $\kappa$ is a $\beta^{*}$-strongly convex function. It holds that if $A$ is full row-rank, then $\beta^{*}$-strong-convexity of $f^{*}$ implies $\alpha$-strong-convexity of $f^{*}\left(-A^{T} \nu\right)$, where $\alpha=\lambda \beta^{*}$ and $\lambda=\lambda_{\min }\left(A A^{T}\right)$ is the minimum eigenvalue of $A A^{T}$. Since $A$ is full row-rank, it follows that $\lambda>0$. Finally, using the fact that $f_{1}=f^{*}\left(-A^{T} \nu\right)$ is $\alpha$-strongly convex and $f_{2}=\nu^{T} b$ is convex, one obtains that $f_{1}+f_{2}=f^{*}\left(-A^{T} \nu\right)+\nu^{T} b=-g(\nu)$ is $\alpha$-strongly convex, or equivalently, $g$ is $\alpha$-strongly concave.

\section{APPENDIX D \\ PROOF OF LEMMA 9}

Proof: Define $H_{x x}=\nabla_{x x} F, H_{x z}=\nabla_{x z} F$ and $H_{z z}=$ $-\nabla_{z z} F$. Since $F$ is twice-continuously differentiable, one has that $\nabla_{z x} F=\left(\nabla_{x z} F\right)^{T}$. Define $H=\nabla^{2} F(x, z)$ so that $H=$ $\left[\begin{array}{cc}H_{x x} & H_{x z} \\ H_{x z}^{T} & -H_{z z}\end{array}\right]$. Note that $H_{x x}$ and $H_{z z}$ are positive definite for all $(x, z) \in U$ due to Assumption 6. The rank of the matrix $H$ satisfies ( [42])

$$
\operatorname{rank} H=\operatorname{rank} H_{x x}+\operatorname{rank}\left(-H_{z z}-H_{x z}^{T} H_{x x}^{-1} H_{x z}\right) .
$$

Now, since $H_{x x}$ is invertible for all $(x, z) \in U$, one has that $\operatorname{rank} H_{x x}=n$. Let $H_{1}=H_{x x}$ and $H_{2}=-H_{z z}-$ $H_{x z}^{T} H_{x x}^{-1} H_{x z}$. Since $H_{x x}, H_{z z}$ are positive definite matrices, it follows that $H_{2}$ is also negative definite. Hence, one obtains that $\operatorname{rank} H_{2}=m$. This implies that $\operatorname{rank} H=\operatorname{rank} H_{1}+$ rank $H_{2}=n+m$ for all $(x, z) \in U$, i.e., $\nabla^{2} F(x, z)$ is full rank and hence, invertible for all $(x, z) \in U$. 